\title{
The Kepler Light Curve of V344 Lyrae: Constraining the Thermal-Viscous Limit Cycle Instability
}

\author{
J. K. Cannizzo ${ }^{1,2}$, M. D. Still ${ }^{3,4}$, S. B. Howell ${ }^{5}$, M. A. Wood $^{6}$, and A. P. Smale ${ }^{2}$
}

\begin{abstract}
We present time dependent modeling based on the accretion disk limit cycle model for a $270 \mathrm{~d}$ light curve of the short period SU UMa-type dwarf nova V344 Lyr taken by Kepler. The unprecedented precision and cadence (1 minute) far surpass that generally available for long term light curves. The data encompass two superoutbursts and 17 normal (i.e., short) outbursts. The main decay of the superoutbursts is nearly perfectly exponential, decaying at a rate $\sim 12 \mathrm{~d}$ $\mathrm{mag}^{-1}$, while the much more rapid decays of the normal outbursts exhibit a fasterthan-exponential shape. Our modeling using the basic accretion disk limit cycle can produce the main features of the V344 Lyr light curve, including the peak outburst brightness. Nevertheless there are obvious deficiencies in our model light curves: (1) The rise times we calculate, both for the normal and superoutbursts, are too fast. (2) The superoutbursts are too short. (3) The shoulders on the rise to superoutburst have more structure than the shoulder in the observed superoutburst and are too slow, comprising about a third to half of the total viscous plateau, rather than the $\sim 10 \%$ observed. However, one of the $\alpha_{\text {cold }} \leftrightarrow \alpha_{\text {hot }}$ interpolation schemes we investigate (one that is physically motivated) does yield longer superoutbursts with suitably short, less structured shoulders.
\end{abstract}

Subject headings: accretion, accretion disks - binaries: close - cataclysmic variables - stars: dwarf novae

\footnotetext{
${ }^{1}$ CRESST/Joint Center for Astrophysics, University of Maryland, Baltimore County, Baltimore, MD 21250, John.K.Cannizzo@nasa.gov

${ }^{2}$ Astroparticle Physics Laboratory, NASA-Goddard Space Flight Center, Greenbelt, MD 20771

${ }^{3}$ Bay Area Environmental Research Inst., Inc., 560 Third St. W, Sonoma, CA 95476, Martin.D.Still@nasa.gov

${ }^{4}$ NASA-Ames Research Center, Moffet Field, CA 94035

${ }^{5}$ National Optical Astronomy Observatory, Tucson, AZ 85719

${ }^{6}$ Department of Physics and Space Sciences, Florida Institute of Technology, 150 W. University Blvd., Melbourne, FL 32901
} 


\section{Introduction}

Dwarf novae constitute a subclass of the cataclysmic variables (Warner 1995b), semidetached interacting binaries in which a Roche-lobe filling secondary transfers matter to a more massive white dwarf (WD). The transferred material possesses angular momentum, and therefore can only "fall" toward the primary down to a radius determined by its specific angular momentum (Lubow \& Shu 1975). This angular momentum barrier is overcome by angular momentum transport within the accretion disk which carries angular momentum outward and mass inward. Current thinking about the physical mechanism responsible for accretion centers on the magneto-rotational instability (=MRI; Balbus \& Hawley 1998) in which the shearing amplification of a weak seed magnetic field leads to turbulent transport.

V344 Lyr is a short period dwarf nova, of subtype SU UMa $\left(P_{\text {orb }}=2.1 \mathrm{~h}\right.$; Still et al. 2010) exhibiting a supercycle pattern (109.6 d; Kato et al. 2002) consisting of superoutbursts separated by normal outbursts. Superoutbursts are long outbursts with slow, exponential decays exhibiting superhumps - modulations in the light curve at a period greater than the

orbital period by a few percent. The data presented in Kato et al. reveal only the brightest portions of the superoutbursts; the fainter portions and all of the normal outbursts lie below the lower limit of detection.

The Kepler data set for V344 Lyrae (Kepler ID 7659570) - 270 d at 1 min cadence - provides an extraordinary resource for accretion disk modelers. The accretion disk limit cycle mechanism, which is currently employed to account for dwarf nova outbursts, is based on the storage of material during quiescence in a non-steady-state configuration, followed by a dumping of matter onto the accreting WD when a critical surface density is attained in the disk, producing an outburst. During outburst the disk is roughly in steady state, except for the action of a cooling front that begins at the outer disk and moves inward. The thermal-viscous limit cycle model is not a complete model for the accretion disk, but rather primarily a model for the outbursts; for instance it fails to account for the high X-ray luminosities seen in dwarf novae in quiescence (Wheatley, Mauche, \& Mattei 2003, Mukai, Zietsman, \& Still 2009).

Previous attempts at constraining physical parameters of the model have relied on AAVSO (American Association of Variable Star Observers) and RASNZ (Royal Astronomical Society of New Zealand) data for the long term light curves. These data are typically given as $1 \mathrm{~d}$ means, with large attendant error bars $(\sim 0.3-0.5 \mathrm{mag})$. The Kepler data provide an entirely new level of precision, and allow detailed model constraints. The viscosity parameter $\alpha$ (Shakura \& Sunyaev 1973) sets the disk time scales. To first order, $\alpha_{\text {cold }}$ controls the recurrence time for outbursts, and $\alpha_{\text {hot }}$ sets the duration of the outbursts. Previous efforts, for instance by Smak (1984=S84), utilized the "Bailey relation" relating the rate of the fast 
decay in DNe with orbital period to infer that $\alpha_{\text {hot }} \simeq 0.1-0.2$. This remains the only firm constraint we have in astronomy for $\alpha$ in ionized gas, and has served as a benchmark value for modelers calculating the efficiency of the MRI in accretion disks (e.g., Hirose et al. 2009). By comparing theoretical modeling of the MRI with the observational constraints, King, Pringle, \& Livio (2007) showed that theoretical values tend to be at least an order of magnitude smaller. Most of the simulations have studied the growth and subsequent nonlinear saturation of the MRI using idealized "shearing boxes" without realistic vertical structure, and for such calculations the asymptotic strength of the magnetic field decreases with increasing resolution (Fromang \& Papaloizou 2007). Vishniac (2009) argues that current models are not sophisticated enough in terms of their dynamic range and treatment of the vertical disk structure to allow a realistic treatment of such important effects as magnetic buoyancy and vertical transport of magnetic helicity by turbulent eddies, and therefore comparisons of theoretical $\alpha$ values with those inferred from observations are specious at the present time.

Our goal in this work is to find model parameters that can account for the gross properties of the long term V344 Lyr light curve. There will be additional features in the light curve that can be compared to the detailed model light curves in order to gain insight into shortcomings of the model. We also investigate systematic effects such as the number of grid points and time step size.

In $\S 2$ we present an overview of the physics of accretion disks. In $\S 3$ we discuss the V344 Lyr light curve, in $\S 4$ we present a review of viscous decays in several well-studied dwarf novae, in $\S 5$ we detail the numerical model, in $\S 6$ we present the results, in $\S 7$ we discuss the results in a larger context, and in $\S 8$ we sum up.

\section{Accretion Disk Physics}

\subsection{Axisymmetric Models}

In the accretion disk limit cycle model, gas accumulates in quiescence and accretes onto the central object in outburst (e.g., Cannizzo 1993a, Lasota 2001 for reviews). The phases of quiescence and outburst are mediated by the action of heating and cooling fronts that traverse the disk and bring about phase transitions between low and high states, consisting of neutral and ionized gas, respectively. During quiescence, when the surface density $\Sigma(r)$ at some radius within the disk exceeds a critical value $\Sigma_{\max }(r)$, a transition to the high state is initiated; during outburst, when $\Sigma(r)$ drops below a different critical value $\Sigma_{\min }(r)$, a transition to the low state is initiated. Low-to-high transitions can begin at any radius, 
whereas high-to-low transitions begin at the outer disk edge. This situation comes about because in the outburst disk $\Sigma(r) \propto r^{-3 / 4}$ (roughly), and the critical surface densities both increase with radius. Since the disk mass accumulated in quiescence is bounded by $\Sigma_{\max }(r)$ and $\Sigma_{\min }(r)$, one can define a maximum disk mass

$$
M_{\text {disk,max }}=\int 2 \pi r d r \Sigma_{\max }(r)
$$

and a minimum disk mass

$$
M_{\text {disk }, \min }=\int 2 \pi r d r \Sigma_{\min }(r)
$$

which will bound the general, time dependent disk mass.

For normal, "short" outbursts, only a few percent of the stored gas accretes onto the central object: the thermal time scale of a thin disk is short compared to the viscous time scale, and the cooling front that is launched from the outer edge of the disk almost as soon as the disk enters into outburst traverses the disk and reverts it back to quiescence. For disks that have been "filled" to a higher level with respect to $M_{\text {disk,max }}$, the surface density in the outer disk can significantly exceed the critical surface density $\Sigma_{\min }$. In order for the cooling front to begin, however, the outer surface density $\Sigma\left(r_{\text {outer }}\right)$ must drop below $\Sigma_{\text {min }}\left(r_{\text {outer }}\right)$. Disks in this state generate much longer outbursts, with slower "viscous" plateaus, because the entire disk must remain in its high, completely ionized state until enough mass has been lost onto the WD for the condition $\Sigma\left(r_{\text {outer }}\right)<\Sigma_{\min }\left(r_{\text {outer }}\right)$ to be satisfied. The slow decay associated with a superoutburst is a direct reflection of the viscous time scale in the outer disk, whereas the subsequent faster decay reveals the thermal time scale. Although the decays of dwarf novae at a given orbital period are fairly uniform, and form the basis for the Bailey relation, the rise times show a greater variety, reflecting the fact that the outburst can be triggered anywhere in the disk (Cannizzo 1998b). Inside-out bursts tend to produce slow-rise times, whereas outside-in bursts produce fast rises (Cannizzo, Wheeler, \& Polidan 1986). Van Paradijs (1983) undertook a statistical study of outburst properties of dwarf novae spanning a range in orbital period, from $<2 \mathrm{hr}$ to $\sim 10 \mathrm{hr}$. By studying separately the behavior of long and short outbursts versus orbital period, he found that the apparent strong contrast between superoutbursts and normal outbursts in the SU UMa systems is due to the fact that the widths of the short (narrow) outbursts scales with orbital period, whereas the widths of the long (wide) outbursts is relatively constant. Van Paradijs' argument is strengthened by the more extensive data set examined by Ak, Ozkan, \& Mattei (2002) in which the linear trend in the narrow width- $P_{\text {orb }}$ relation is more obvious (compare Fig. 4 from Ak et al. with Fig. 3b from van Paradijs). In these studies the widths of the outbursts were "corrected" by subtracting the rise and decay times.

For any dynamical system in which a variety of timescales contribute to a physical 
observable, in this case the flux from the accretion disk, the controlling time scale will be the slowest one. Since all the disk timescales - dynamical, thermal, and viscous - increase with radius, the time scale associated with the viscous plateau provides direct information about the viscous time scale $t_{\text {visc }}$ at the outer edge of the disk.

Warner (1995ab) presents a simple physical model for the state of the disk during superoutburst. During the superoutburst the disk is roughly in steady state with $\Sigma(r) \propto$ $r^{-3 / 4}$ out to some $r_{d}$ which represents the radius at which the outward moving heating front stalls. The rate of accretion within the disk is much greater than the mass transfer rate from the secondary star, which can be neglected during this time. Therefore the mass of the hot, accreting disk is

$$
M_{\mathrm{disk}} \approx \frac{8 \pi}{3} r_{d}^{2} \Sigma_{\min }\left(r_{d}\right)
$$

From the standard accretion disk theory relating effective temperature with accretion rate in a steady-state disk (Shakura \& Sunyaev 1973), one has

$$
\dot{M}_{\mathrm{disk}}=-\frac{8 \pi \sigma}{3 G M_{1}} T_{\mathrm{eff}}^{4}\left(r_{d}\right) r_{d}^{3}
$$

where $T_{\text {eff }}\left(r_{d}\right)=T_{\text {eff }}\left(\Sigma_{\min }\left[r_{d}\right]\right)$ is the effective temperature associated with $\Sigma_{\min }\left(r_{d}\right)$. Combining eqns. 3] and [4 (same as eqns. [15] and [16] from Warner 1995a, and [3.46] and [3.47] from Warner 1995b) yields

$$
\frac{d M_{\mathrm{disk}}}{M_{\mathrm{disk}}} \approx-\frac{5 r_{d} \sigma T_{\mathrm{eff}}^{4}\left(r_{d}\right)}{3 G M_{1} \Sigma_{\min }\left(r_{d}\right)} d t .
$$

Since the right hand side (excluding $d t$ ) is constant, the solution for the mass of the hot disk, and also its derivative, is a decreasing exponential function. Using power law scalings for $T_{\text {eff }}\left(r_{d}\right)$ and $\Sigma_{\min }\left(r_{d}\right)$, setting $r_{d}$ equal to the 3:1 resonance radius with the binary orbital period, and noting that luminosity varies as $\dot{M}_{\text {disk }}$, one can show (cf. eqn. [20] of Warner 1995a)

$$
t_{\mathrm{visc}} \approx 17 \mathrm{~d} \alpha_{-1}^{-4 / 5} P_{h}^{1 / 4} m_{1}^{1 / 6}
$$

where $\alpha_{-1}=\alpha / 0.1, P_{h}$ is the orbital period in hr, and $m_{1}=M_{1} / M_{\odot}$. (The $\alpha$ in this context is the hot state value.) Within the viscous plateau portion of a superoutburst, the instigation and propagation of a cooling front at the outer edge is thwarted by virtue of excessive mass in the disk. This phenomenon is not new and has been seen in numerous previous studies (e.g., Cannizzo 1993b=C93b, Hameury et al. 1998=H98, Ludwig \& Meyer 1998, Buat-Ménard, Hameury, \& Lasota 2001, Schreiber, Hameury, \& Lasota 2003). The viscous time does not represent the duration of the outburst itself, but rather the time scale (approximately in $\mathrm{d}$ $\operatorname{mag}^{-1}$ ) associated with the viscous plateau; it represents an $e$-folding time for the decrease of disk mass. 


\subsection{Non-axisymmetric Models}

Whitehurst (1988) discovered a hydrodynamical instability that can occur in the outer parts of accretion disks when the mass ratio $q=M_{2} / M_{1}$ is less than $1 / 4$. For these extreme mass ratio systems, expected in CVs below the period gap if the secondary fills its Roche lobe, the outer part of the disk can lie beyond the point of 3:1 resonance with the binary period. The outer disk can then be excited by the inner Lindblad resonance (Lubow 1991ab), causing the global disk oscillation mode that is observed as common superhumps. Before the onset of the superhump oscillation, there are well-known spiral dissipation waves in the disk that are fixed in the co-rotating frame. Once the superhump oscillation begins, these spiral arms advance $180^{\circ}$ every superhump cycle 7 . As a given arm passes between the two stars, it expands outward in the shallower potential, but as fluid in that arm compresses back into the disk as the disk is maximally distorted, viscous dissipation causes a peak in the photometric light curve (Simpson \& Wood 1998). A secondary source of the superhump signal is caused by the variable distance the mass stream must fall from the L1 point to the edge of the non-axisymmetric, flexing disk. Because the outer disk is thought to expand during superoutburst, potentially beyond the 3:1 radius, the "tidal instability" model provides a natural explanation for superhumps. If the mass ratio is extreme enough, superhumps can also occur outside of superoutbursts because the disk can remain extended beyond the 3:1 radius, and therefore continue oscillating even into quiescence. Osaki (1989ab) combined the accretion disk limit cycle model with Whitehurst's tidal instability model and proposed the "thermal-tidal" instability model (TTIM) for superoutbursts. This model posits that the presence of an oscillating precessing disk, triggered by the migration of gas beyond the 3:1 resonance radius, also leads to a greatly enhanced tidal torque acting on the disk. This ultimately contracts the outer disk and leads to a superoutburst. Thus in the TTIM one has the usual hysteresis relation between surface density and temperature in the disk, and also a hysteresis relation between the total angular momentum of the disk and the tidal torque acting on the outer disk. (The latter hysteresis is somewhat speculative.) This model was examined in detail and compared to the enhanced mass transfer model (EMTM) for superoutbursts by Ichikawa, Hirose, \& Osaki (1993) and later workers, most recently Schreiber et al. (2004).

For completeness, we note that Smak has recently challenged the conventional wisdom that superhumps are due to a precessing, eccentric disk (Smak 2009abcd). Smak (2009a) presents a re-analysis of data taken by earlier workers on Z Cha, WZ Sge, OY Car, and IY UMa and claims that a variety of errors, including miscalculated beat phases and an

\footnotetext{
${ }^{7}$ See, for example, www.astro.fit.edu/wood/visualizations.html.
} 
assumption that observed eclipses are pure disk eclipses, led to erroneously large values of the eccentricity $e$. Smak's re-analysis of the disk eccentricity in Z Cha leads to $e=0.05 \pm$ 0.05. Smak (2009b) compares in detail observed values for the amplitudes of superhumps with theoretical values determined from bolometric light curves produced by smooth-particle hydrodynamics calculations. He finds the observed values to be $\sim 10$ times larger than the theoretical values. Smak (2009c) presents a re-analysis of five eclipses of Z Cha observed by Warner \& O'Donoghue (1988). Smak examines two local minima at orbital phases $\Phi \approx-0.05$ and 0.04 . He argues that the first minimum is not due to an occultation but rather absorption in the mass overflow stream, and the second minimum coincides with the trajectory of the mass overflow stream. The upshot of his analysis is that superhumps are due to modulated mass transfer which leads to periodically enhanced dissipation of the stream kinetic energy. Smak (2009d) presents evidence for periodically variable irradiation of secondary components and proposes that superhumps are due to enhanced dissipation of the stream kinetic energy. He outlines specific future modeling efforts that might clarify the overall picture.

\section{Kepler light curve of V344 Lyr}

The bandpass utilized by Kepler is considerably broader than the standard Johnson $V$ band. Figure 1 gives a comparison of the two filters 8 . Throughout this paper we will refer to Johnson $V$ apparent and absolute magnitudes using $V$ and $M_{V}$, and Kepler apparent and absolute magnitudes using $K p$ and $M_{\mathrm{Kp}}$. Figure 2 shows the Kepler light curve of V344 Lyr. The observation window contains 19 outbursts showing the sequencing SSSLSSSSSSSLSSSSSSS, where $\mathrm{S}=$ short outburst and L=long outburst, i.e., a superoutburst. Based on photographic observations made by Hoffmeister (1966), Kato (1993) estimated that the cycle length for normal outbursts was $16 \pm 3 \mathrm{~d}$. An estimate of this cycle length prior to Kepler was difficult because the normal outbursts of V344 Lyr are short-lived and reach only to near $K p=16$ (or $V \simeq 17$ ). In Table 1 we list the durations (using $K p=18$ as the cut line) and amplitudes (measured from the local mean quiescence level) of the 19 observed outbursts (17 normal and two superoutbursts). Both the recurrence times and amplitudes of the normal outbursts appear to reach a maximum roughly midway between superoutbursts, rather than increasing monotonically between consecutive superoutbursts as in the TTIM. We also note that the level of quiescence is $\sim 1$ mag higher after the superoutburst, and declines with an $e$-folding time of $\sim 50 \mathrm{~d}$. This trend may be associated with the cooling of the WD after being heated by the mass accreted during superoutburst (e.g., Sion 1995).

\footnotetext{
${ }^{8}$ The Kepler response is taken from http://keplergo.arc.nasa.gov/CalibrationResponse.shtml.
} 
The superoutbursts last $\sim 17 \mathrm{~d}$ and reach a maximum amplitude $\Delta K p=4.4-4.5$ mag. The decline of the superoutbursts are close to linear (plotted as mag versus time, or exponential as flux versus time) for $\sim 11 \mathrm{~d}$, after which they fall rapidly $(\sim 2 \mathrm{~d})$ back to quiescence. During this part the decline rate is $0.083 \mathrm{mag} \mathrm{d}^{-1}$, in good agreement with the value of $0.094 \mathrm{mag} \mathrm{d}^{-1}$ found by Kato (1993). The amount of time spent at $K p \lesssim 16$ is $\sim 14$ $\mathrm{d}$, and the duration of the entire plateau is $\sim 12 \mathrm{~d}$. The decay rate is $\sim 12 \mathrm{~d} \mathrm{mag}^{-1}$, therefore the plateau portion of the decay encompasses $\sim 1 \mathrm{mag}-$ a dynamic range of $\sim 2.5$, or slightly less than one $e$-folding $(\sim 2.7 \times)$. There are also $\sim 0.5 \mathrm{mag}$ shoulders on the superoutburst rises lasting $\sim 1 \mathrm{~d}$, which appear to be normal outbursts embedded at the beginning of the superoutbursts.

It is noteworthy that the fast decays of the normal outbursts and superoutburst have a faster-than-exponential shape (i.e., concave downward when plotted as magnitude versus time). S84 utilized the Bailey relation between the fast rate of decay in dwarf novae and their orbital period to constrain $\alpha_{\text {hot }} \simeq 0.1-0.2$. The large errors associated with the AAVSO data were consistent with exponential decay. Cannizzo (1994) found that to account for the supposedly exponential decays, which correspond to the time during which a cooling front traverses the disk, $\alpha$ must vary weakly with radius $\left(\tilde{\propto} r^{0.3}\right)$. The faster-than-exponential decays captured by Kepler in V344 Lyr negate this result and indicate that a constant $\alpha$ scaling with radius is consistent with the data.

\section{Viscous Plateaus in Dwarf Novae}

The Bailey relation between the fast rate of decay of dwarf nova outbursts and orbital period corresponds to the time interval during which the cooling front traverses the disk. Some long dwarf nova outbursts have enough dynamic range in $V$ that one can also use the slow decay portion, the viscous plateau, to infer an $\alpha$ value. These inferred values agree with those determined from the Bailey relation (Warner 1995ab; Cannizzo 2001b, Cannizzo et al. 2002).

The decay rates associated with several dwarf novae exhibiting viscous plateaus have been fairly well established, and enable one to make a reasonable estimate of the decay rate expected in a system with the orbital period of V344 Lyr. The Kepler observation gives a decay rate within the superoutburst $\sim 12 \mathrm{~d} \mathrm{mag}^{-1}$. We associate this with the viscous time, which is basically an $e$-folding time for a perturbation to the $\Sigma(r)$ profile to be damped out. For a disk with a $\Sigma(r)$ profile approximating steady state, i.e., $\Sigma(r) \propto r^{-3 / 4}$, and for which the mass flow within the disk and onto the WD greatly exceeds the rate of mass addition

feeding into the outer disk from the secondary star, the viscous time represents roughly a 
time for the mass of the disk to decrease by a factor $e$.

Thus for a given viscous plateau-type outburst, the ratio $t_{\text {plateau }} / t_{\text {visc }}$ represents the number of $e$-foldings by which the mass of the disk decreases due to accretion onto the central object. It is instructive to look at examples of viscous decays in several well-studied dwarf novae:

SS Cyg ${ }^{9}$ : Long outbursts in SS Cyg $\left(P_{\text {orb }}=6.6 \mathrm{hr}\right.$ ) have a duration of $\sim 10 \mathrm{~d}$ (Cannizzo \& Mattei 1992). and the viscous time in the outer disk $t_{\mathrm{visc}} \approx 40 \mathrm{~d}$. Thus $t_{\text {plateau }} \ll t_{\mathrm{visc}}$, and only about $20 \%$ of the disk mass is accreted onto the WD (C93b).

U Gem: In the 1985 long outburst of U Gem $\left(P_{\text {orb }}=4.25 \mathrm{hr}\right)$, the decay rate during the viscous plateau was $\sim 26 \mathrm{~d} \mathrm{mag}^{-1}$ over the $\sim 35 \mathrm{~d}$ of the burst (Cannizzo et al. 2002). Since $t_{\mathrm{visc}} \approx t_{\text {plateau }}, \sim 70 \%$ of the disk mass accreted onto the WD.

V344 Lyr: In the superoutbursts of V344 Lyr $\left(P_{\text {orb }}=2.1 \mathrm{hr}\right)$ shown in Fig. 2 the locally defined decay rate during the plateau remains constant at $\sim 12 \mathrm{~d} \mathrm{mag}^{-1}$ over most of the $\sim 14 \mathrm{~d}$ of the burst, thus as with $\mathrm{U}$ Gem $\sim 70 \%$ of the disk mass accreted onto the WD.

WZ Sge: In the 2001 superoutburst of WZ Sge $\left(P_{\text {orb }}=81 \mathrm{~min}\right)$, the locally defined decay rate during the plateau increased from $\sim 4$ to $\sim 12 \mathrm{~d} \mathrm{mag}^{-1}$ over the $\sim 20 \mathrm{~d}$ of the burst (Cannizzo 2001b). The fact that $t_{\text {plateau }} \approx 3 t_{\text {visc }}$ means that only a few percent of the initial disk mass remained at the end of the superoutburst. (An alternative considered in the Discussion is that irradiation-induced enhanced mass overflow from the secondary star prolonged the superoutburst.) Without a very low $\alpha_{\text {cold }} \simeq 10^{-5}$ there is not enough accumulated mass in the disk to account for the outburst's fluence (Smak 1993) and the long recurrence time.

In summary, one can readily find examples of known systems with viscous type outbursts in which the duration of the outburst itself is either greater than, less than, or about the same as the viscous time scale evaluated at the outer edge of the accretion disk. The actual course taken by a given system depends on the degree of overfilling of the disk in quiescence with respect to $M_{\text {disk,min }}$.

\footnotetext{
${ }^{9}$ If one accepts the HST/FGS trigonometric parallax for SS Cyg 6.02 \pm 0.46 mas (Harrison et al. 1999) which implies $D=166 \pm 13 \mathrm{pc}$, the accretion disk limit cycle model is not able to reproduce dwarf nova outbursts for SS Cyg (Schreiber \& Lasota 2007, Smak 2010). Harrison et al. (2004) acquire spectra of MK standard stars to refine the mean absolute parallax of the reference frame and present a reanalysis of the SS Cyg parallax (see their sect. 2.3). They obtain a revised value $6.06 \pm 0.44$ mas - close to their original value.
} 


\section{Numerical Modeling}

Our numerical model is discussed extensively in C93b and subsequent papers. The basic strategy is the time dependent solution of two coupled differential equations, one giving the $\Sigma(r)$ evolution, and one the evolution of disk midplane temperature $T(r)$. Thus the disk is entirely unconstrained as regards deviations from steady state and thermal equilibrium. Many previous workers have presented time dependent calculations by solving these equations, or similar versions (e.g., S84; Lin, Papaloizou, \& Faulkner 1985=L85; Mineshige \& Osaki 1983=M83, 1985=M85, Mineshige 1986=M86, 1987=M87; Pringle, Verbunt \& Wade 1986=P86; Cannizzo et al. 1986=C86, C93b, 1994, 1998a, 2001a; Angelini \& Verbunt 1989; Ichikawa \& Osaki 1992; Meyer \& Meyer-Hofmeister 1984, Ludwig \& Meyer 1998; H98, Menou, Hameury, \& Stehle 1999, Buat-Ménard, Hameury, \& Lasota 2001, Buat-Ménard, \& Hameury 2002, Schreiber, Hameury, \& Lasota 2003, 2004=S04).

Improvements in our code made since the original version include the provision for a variable outer disk radius, self-irradiation, and evaporation with various possible laws (e.g., Meyer \& Meyer-Hofmeister 1994). The diffusion equation governing the evolution of surface density is given by (Lightman 1974, Pringle 1981)

$$
\frac{\partial \Sigma}{\partial t}=\frac{3}{r} \frac{\partial}{\partial r}\left[r^{1 / 2} \frac{\partial}{\partial r}\left(\nu \Sigma r^{1 / 2}\right)\right],
$$

where $\Sigma(r, t)$ is the surface density and $\nu$ is the kinematic viscosity coefficient,

$$
\nu=\frac{2}{3} \frac{\alpha}{\Omega} \frac{\mathcal{R} T}{\mu} .
$$

We follow the technique of Bath \& Pringle (1981) in discretizing the $\Sigma(r, t)$ evolution equation.

The thermal energy equation governing the evolution of the midplane disk temperature $T(r, t)$ is given by

$$
\frac{\partial T}{\partial t}=\frac{2(A-B+C+D)}{c_{p} \Sigma}-\frac{\mathcal{R} T}{\mu c_{p}} \frac{1}{r} \frac{\partial}{\partial r}\left(r v_{r}\right)-v_{r} \frac{\partial T}{\partial r},
$$

where the viscous heating

$$
A=\frac{9}{8} \nu \Omega^{2} \Sigma,
$$

the radiative cooling

$$
\begin{gathered}
B=\sigma T_{e}^{4}, \\
C=\frac{3}{2} \frac{1}{r} \frac{\partial}{\partial r}\left(c_{p} \nu \Sigma r \frac{\partial T}{\partial r}\right),
\end{gathered}
$$


and

$$
D=\frac{h}{r} \frac{\partial}{\partial r}\left(r \frac{4 a c T^{3}}{3 \kappa_{R} \rho} \frac{\partial T}{\partial r}\right) .
$$

Of the four terms appearing in the numerator of the first term of thermal energy equation, the first two $A$ and $B$ are fairly standard. The term $C$ represents the radial heating flux due to turbulent transport, and $D$ is that due to radiative transport. Previous workers have used varying forms of $C$ and $D$. Some early studies adopted $C=D=0$ (M83), $C=0$ (L85), or a form of $C$ not expressible as the divergence of a flux (M83, M86, C93b; see H98 for a critical discussion).

The local flow velocity $v_{r}$, which can vary enormously from inflow to outflow over the radial extent of the disk, depending on whether or not transition fronts are present, is given by

$$
v_{r}=-\frac{3}{\Sigma r^{1 / 2}} \frac{\partial}{\partial r}\left(\nu \Sigma r^{1 / 2}\right)
$$

where upwind differencing is implemented over the radial grid as follows:

$$
\begin{aligned}
& s_{a}=\nu_{i-1} \Sigma_{i-1} r_{i-1}^{1 / 2} \\
& s_{b}=\nu_{i} \Sigma_{i} r_{i}^{1 / 2} \\
& s_{c}=\nu_{i+1} \Sigma_{i+1} r_{i+1}{ }^{1 / 2} \\
& q_{-}=-\frac{3}{\sum_{i} r_{i}^{1 / 2}} \frac{s_{b}-s_{a}}{r_{i}-r_{i-1}} \\
& q_{+}=-\frac{3}{\sum_{i} r_{i}^{1 / 2}} \frac{s_{c}-s_{b}}{r_{i+1}-r_{i}} \\
& \text { if }\left(\frac{q_{-}}{q_{+}}>0 \text { and } q_{-}>0\right) \quad\left(v_{r}\right)_{i}=\max \left(q_{-}, q_{+}\right) \\
& \text {if }\left(\frac{q_{-}}{q_{+}}>0 \text { and } q_{-}<0\right) \quad\left(v_{r}\right)_{i}=\min \left(q_{-}, q_{+}\right) \\
& \text {if }\left(\frac{q_{-}}{q_{+}}<0 \text { and } \frac{\left|q_{-}\right|}{\left|q_{+}\right|}>1\right) \quad\left(v_{r}\right)_{i}=q_{-} \\
& \text {if }\left(\frac{q_{-}}{q_{+}}<0 \text { and } \frac{\left|q_{-}\right|}{\left|q_{+}\right|}<1\right) \quad\left(v_{r}\right)_{i}=q_{+} \text {. }
\end{aligned}
$$


The scalings for the local maximum and minimum in the $\Sigma-T$ relation (from Meyer \& Meyer-Hofmeister 1981, 1982) are

$$
\Sigma_{\max }=444 \mathrm{~g} \mathrm{~cm}^{-2} r_{10}^{1.1} m_{1}^{-0.37} \alpha_{c,-2}^{-0.7},
$$

where $\alpha_{c,-2}=\alpha_{\text {cold }} / 0.01$, and

$$
\Sigma_{\min }=44.4 \mathrm{~g} \mathrm{~cm}^{-2} r_{10}^{1.1} m_{1}^{-0.37} \alpha_{h,-1}^{-0.7}
$$

where $\alpha_{h,-1}=\alpha_{\text {hot }} / 0.1$. The disk midplane temperatures associated with these extrema are

$$
T\left(\Sigma_{\max }\right)=5275 \mathrm{~K} \alpha_{c,-2}^{-0.3}
$$

and

$$
T\left(\Sigma_{\min }\right)=35900 \mathrm{~K} r_{10}^{0.064} m_{1}^{-0.02} \alpha_{h,-1}^{-0.16}
$$

The equilibrium temperature scalings for the stable branches are given in C93b (taken from Cannizzo \& Wheeler 1984). During transitions when the local disk temperature lies between the values associated with the local maximum and minimum in surface density, $T\left[\Sigma_{\max }(r)\right]<$ $T(r)<T\left[\Sigma_{\min }(r)\right]$, we use a logarithmic interpolation to obtain the local $\alpha$ value,

$$
\log _{10} \alpha(r)=\log _{10} \alpha_{\text {cold }}+f
$$

where

$$
f=\frac{T-T\left(\Sigma_{\max }\right)}{T\left(\Sigma_{\min }\right)-T\left(\Sigma_{\max }\right)}\left(\log _{10} \alpha_{\text {hot }}-\log _{10} \alpha_{\text {cold }}\right) .
$$

In tests we also consider a logarithmic interpolation factor $f$ introduced by H98,

$$
f=\left[1+\left(\frac{T_{0}}{T}\right)^{8}\right]^{-1}\left(\log _{10} \alpha_{\text {hot }}-\log _{10} \alpha_{\text {cold }}\right),
$$

as well as a linear interpolation

$$
\alpha(r)=\alpha_{\text {cold }}+\frac{T-T\left(\Sigma_{\max }\right)}{T\left(\Sigma_{\min }\right)-T\left(\Sigma_{\max }\right)}\left(\alpha_{\text {hot }}-\alpha_{\text {cold }}\right) .
$$

In calculating the effects of the tidal torque from the secondary we follow Smak (1984) and later workers who used a formalism developed by Papaloizou \& Pringle (1977) in which the tidal torque varies as the fifth power of radius. This leads to a depletion in disk material

$$
\frac{\partial \Sigma}{\partial t}=-c_{1} \omega \frac{\nu \Sigma}{2 \pi j_{s}}\left(\frac{r}{a}\right)^{5}
$$


where $\omega$ is the orbital angular frequency $2 \pi / P_{\text {orb }}$, the orbital separation is $a$, and the specific angular momentum $j_{s}=\left(G M_{1} r\right)^{1 / 2}$. We do not reset the value of $c_{1}$ during the course of a run as in the TTIM. Ichikawa \& Osaki (1994) presented detailed calculations of the strength of the tidal torque versus distance at large radii within the disk and found that the power law $\left(\propto r^{5}\right)$ determined by Papaloizou \& Pringle is only valid at one radial location in the outer disk; further out the torque relation steepens considerably, approaching infinite slope at the last non-intersecting orbit. Therefore in some sense the more naive "brick wall" condition for the outer edge used by C93b and other workers might be more descriptive. Regardless of which prescription is used, if the parameters associated with the outer edge are not manipulated during the run, the detailed treatment is not important to the overall model. Using a different end point treatment would result in slightly different optimal parameters.

Given a disk temperature $T$ and surface density $\Sigma$, the disk semithickness $h$ and density $\rho$ are determined from vertical hydrostatic equilibrium to yield

$$
h=\frac{P_{r}+\sqrt{P_{r}^{2}+c_{2} c_{3}}}{c_{2}},
$$

where

$$
\begin{gathered}
c_{2}=\Sigma \Omega^{2}, \\
c_{3}=\Sigma \frac{\mathcal{R} T}{\mu},
\end{gathered}
$$

the radiation pressure

$$
P_{r}=\frac{1}{3} a T^{4}
$$

and the density

$$
\rho=\frac{\Sigma}{2 h}
$$

To determine the computational time step, we take

$$
\Delta t=f_{t} \min \left[\min _{i}\left(\frac{\partial \ln \Sigma_{i}}{\partial t}\right)^{-1}, \min _{i}\left(\frac{\partial \ln T_{i}}{\partial t}\right)^{-1}\right],
$$

where $\min _{i}$ refers to the minimum over all grid points $i$, and $f_{t}$ is a small number between $1 / 80$ and $1 / 640$. The quantities $\partial \ln \Sigma_{i} / \partial t$ and $\partial \ln T_{i} / \partial t$ are evaluated using the evolution equations.

\section{Results}

To calculate the long-term light curves from our numerical trials, we assume local Planckian flux distributions for each annulus, and in each time step integrate over the radial 
profiles of effective temperature. We compute the absolute Kepler magnitude $M_{\mathrm{Kp}}$, using the filter shown in Fig. 1, assuming a face-on disk. To account for the background contribution, assumed constant, we add the secondary star and hot spot. For the secondary, we assume an $\mathrm{M} 5 \mathrm{~V}$ star with radius $0.221 R_{\odot}=1.54 \times 10^{10} \mathrm{~cm}$ and $T_{\text {eff }}=2951 \mathrm{~K}$. This contributes $M_{\mathrm{Kp}}=11.31$. For the hot spot, assuming a $12000 \mathrm{~K}$ emitting circular region with radius $10^{9} \mathrm{~cm}$ gives $M_{\mathrm{Kp}}=10.41$. As a test of our magnitude subroutine, using solar parameters $R_{\odot}=6.96 \times 10^{10} \mathrm{~cm}$ and $T_{\text {eff }}=5778 \mathrm{~K}$ we find $M_{V}=4.85$ (the accepted value is 4.83 ) and $M_{\mathrm{Kp}}=3.83$. (The $M_{V}$ values for our putative secondary and hot spot are 12.97 and 11.30, respectively.) A reasonable inclination $i \simeq 45^{\circ}$ would dim the disk by $\sim 0.4$ mag.

After some experimentation, we found a set of parameters that reproduces approximately the overall pattern of supercycle and short outbursts. We take a central $0.6 M_{\odot}$ WD accretor, an inner disk radius $r_{\text {in }}=2 \times 10^{9} \mathrm{~cm}$, and a maximum outer disk radius $r_{\text {out }}=10^{10} \mathrm{~cm}$, a mass feeding rate into the outer disk $\dot{M}_{T}=2 \times 10^{-10} M_{\odot} \mathrm{yr}^{-1}$, and $\alpha$ values $\alpha_{\text {cold }}=0.0025$ and $\alpha_{\text {hot }}=0.1$. The recurrence time for outbursts varies inversely with $\dot{M}_{T}$ and $\alpha_{\text {cold }}$, and the duration of outbursts varies inversely with $\alpha_{\text {hot }}$ (Cannizzo, Shafter \& Wheeler 1988=CSW, C93b). Also, the ratio of the number of short to long outbursts in a long sequence varies in a complicated way on these parameters, and on $r_{\text {in }}$ and $r_{\text {out }}$. The strengths of the dependencies of the various time scales - recurrence, burst duration, rise, and decay - on the input parameters vary according to location within the multidimensional $\left(\dot{M}_{T}, \alpha_{\text {cold }}, \alpha_{\text {hot }}, r_{\text {in }}, r_{\text {out }}, m_{1}\right)$ parameter space (CSW, C93b).

We use the $\alpha$ interpolation given by eqn. (24). As regards the inner disk radius, the value for $r_{\text {inner }}$ is larger than the WD itself; if we take $r_{\text {inner }} \simeq r_{\mathrm{WD}}$, we tend to get far fewer short outbursts between two successive superoutbursts. This hint of a large inner radius may have some observational support in the SU UMa systems (e.g., Howell et al. 1999). The ratio $\alpha_{\text {cold }} / \alpha_{\text {hot }}=40$ is about 10 times greater than commonly used in dwarf nova calculations. The mass transfer rate from the secondary $\dot{M}_{T}$ is $\gtrsim 2$ times the nominal value $\sim 0.8 \times 10^{-10} M_{\odot} \mathrm{yr}^{-1}$ found from evolutionary calculations of cataclysmic variables below the period gap where the evolution is driven predominantly by gravitational radiation (e.g., Kolb, King, \& Ritter 1998; Howell, Nelson, \& Rappaport 2001). When one takes into account the $\sim 20 \%$ depletion of the disk mass accompanying each of the short (normal) outbursts, however, the effective rate of accumulation of mass in the disk between successive superoutbursts decreases from $2 \times 10^{-10} M_{\odot} \mathrm{yr}^{-1}$ to $\sim 0.3 \times 10^{-10} M_{\odot} \mathrm{yr}^{-1}$. In our models, $\sim 40-60 \%$ of the disk mass is accreted during a superoutburst.

In using a complicated numerical model, it is important to carry out testing to gain an understanding of systematic effects: 


\subsection{Interpolation between $\alpha_{\text {cold }}$ and $\alpha_{\text {hot }}$}

Figure 3 shows the effect of using a log versus a linear interpolation for the $\alpha$ value between $\alpha_{\text {cold }}$ and $\alpha_{\text {hot }}$. The linear interpolation leads to smaller amplitude outbursts with little or no quiescent intervals. The decays are also much slower, and concave upwards when plotted as mag versus time (i.e., with a functional form that is slower-than-exponential). Figure 4 shows the effect of using the H98 interpolation. H98 adopted the constant $T_{0}=$ $25000 \mathrm{~K}$ in their scaling; we also look at smaller values $18750 \mathrm{~K}$ and $12500 \mathrm{~K}$. The smaller $T_{0}$ values produce superoutbursts which have the recognizable plateau and subsequent fast decay, but for the $T_{0}=12500 \mathrm{~K}$ run the fast decays are slower-than-exponential. Hameury (2002) compared the two cases $T_{0}=24000 \mathrm{~K}$ and $T_{0}=8000 \mathrm{~K}$ and found little difference between the resultant light curves (see his Fig. 3) in his model.

Figure 5 depicts the different interpolations as well as parameterizations of the fractional ionization $\xi$. The MRI is thought to mediate the strength of $\alpha$ through turbulent transport, and the effect of shearing on the weak magnetic field embedded in the gas is more efficient as $\xi$ increases. The $\mathrm{H} 98$ scaling for $T_{0}=12500 \mathrm{~K}$ does a reasonable job in approximating the $\rho=10^{-6} \mathrm{~g} \mathrm{~cm}^{-3}$ curve for $\xi$, and may be more physical than the other scalings.

\subsection{Number of Grid Points $N$}

Early time dependent accretion disk limit cycle studies were concerned mainly with showing the ability of the disk, under the conditions of an imposed limit cycle in each annulus, to undergo collective, global oscillations in which a major part of the disk participated. The total numbers of grid points $N$ was small and the comparison with observational data minimal (e.g., $N=20-25$ : S84; $N=35$ : L85; $N=22-44$ : M85; $N=17$ : M86; $N=40: \mathrm{P} 86 ; N=44: \mathrm{C} 86)$. One can see obvious irregularities in many of the early light curves which are characteristic of numerical instability. C93b undertook the first detailed investigation regarding the number of grid points, and found that at least several hundred were required to obtain reliable light curves. H98 utilized both uniform and adaptive mesh codes to study the properties of the transition fronts, and found a requirement of $N \gtrsim 800$ for a uniform-equivalent mesh to resolve the fronts adequately.

Figure 6 shows a detail of one superoutburst for models with $N=200$ to 1600 grid points. In term of the long term light curves, even with $N>1000$ the sequencing of long and short outbursts is not perfectly stable using our explicit code. An implicit code such as that described in H98 may be more numerically stable. In terms of individual outburst profiles, the rise times we calculate for both the normal and superoutbursts are too fast. In 
addition, our outbursts are too bright by $\sim 0.5$ mag. A nominal inclination $i \simeq 45^{\circ}$ would make our disks dimmer by $\sim 0.4 \mathrm{mag}$, bringing them close to the level observed in outburst. For comparison we also plot the V344 Lyr data, where $D=620 \mathrm{pc}$ was assumed in converting to absolute magnitude (found by Ak et al. 2008 using a period-luminosity relation). There is a slight increase in superoutburst duration from $N=200$ to $N=800$ due to the fact that more mass accumulates as the supercycle lengthens. In addition, the shoulder in the light curve also becomes longer. For the highest $N$ run the duration of the superoutburst is still shorter than that seen in V344 Lyr, while the shoulder is too long. The superoutburst calculated using the H98 scaling with $T_{0}=12500 \mathrm{~K}$ better reproduces the observed duration of the V344 Lyr superoutburst, and also has a shorter shoulder with less structure than the other calculations. However, it suffers from a rise time that is too short, and the fast decay is now slower-than-exponential. Also, the viscous decay rate, which has been optimized to the observed value using the eqn. (24) scaling, is now too fast.

\subsection{Thermal Energy Equation}

Considering the many forms of the thermal energy equation used by previous workers, and the potential limitations of employing a one-dimensional hydrodynamical formalism, we now look at the effects of switching off various terms in the thermal energy equation in an attempt to ascertain the relative importance of each term. Figure 7 shows the effect of setting equal to zero: (i) $C$, (ii) $D$, (iii) $C$ and $D$, (iv) the first advective term in eqn. (9), and (v) the second advective term in eqn. (9). In the $C=0$ run one sees a rounded superoutburst and only three short outbursts between two superoutbursts. The (radiative) $D$ term is less influential, as the superoutburst profile is relatively unchanged from the first panel. Setting $C=D=0$ produces a single dip in the rise to superoutburst, and a longer viscous plateau (i.e., exponential decay). The first advective term appears to be of comparably minimal importance as the $D$ term; i.e., the omission of either has a negligible effect. The second advective term is the most influential of all terms examined: without it one has much longer superoutbursts - about $70 \%$ of the accumulated disk mass is accreted, which is about the same as in V344 Lyr. However, the viscous plateau still comprises less than half of the superoutburst, rather than the $\gtrsim 90 \%$ seen in V344 Lyr.

\subsection{Detuning of Optimal Parameters}

There is a complicated interplay between the different parameters that enter into the overall results. Figure 8 shows the effect of varying the inner disk radius. For smaller inner 
disk radii, one has a larger dynamic range in $r_{\text {outer }} / r_{\text {inner }}$ and as a result there tends to be a more complete emptying of the disk during outbursts, and therefore less of a tendency to have a series of short outbursts leading up to a superoutburst. The net interaction among all the model parameters is such that the observed sequencing for V344 Lyr is roughly reproduced with $r_{\text {inner }} \simeq 2 \times 10^{9} \mathrm{~cm}$. Figure 9 shows the effect of varying the outer disk radius. For larger $r_{\text {outer }}$ values not only does the number of short outbursts increase, but also the shape of the superoutburst distorts significantly from the viscous plateau shape.

\subsection{Relevance of Results to the V344 Lyr light curve}

No one light curve we have shown reproduces all features of the observed light curve shown in Fig. 2. For instance, there is an atypically long quiescent period at the beginning of the observed light curve. The recurrence time for outbursts varies inversely to varying degrees with $\alpha_{\text {cold }}, \alpha_{\text {hot }}$, and $\dot{M}_{T}(\mathrm{CSW})$. Therefore if any one of these parameters were anomalously small, that could delay an outburst. Also, the pre-superoutburst normal outbursts are brighter than the post-superoutburst normal outbursts, and the pre-superoutburst quiescent level is fainter than the post-superoutburst quiescent level. As noted earlier, the disk instability model is ill-equipped to make any statement about the quiescent state. However, one possibility might be that $\dot{M}_{T}$ were elevated for a while after the superoutburst; that could lead to crowding of the short outbursts, and an increase in the quiescent level. There may be other causal relations among $\alpha_{\text {cold }}, \alpha_{\text {hot }}$, and $\dot{M}_{T}$, which are important.

An analysis of the phase variations in the quiescent-state oscillations in the light curve at the beginning of Fig. 2 light curve show these are likely to be negative superhumps. As such they indicate accretion onto an accretion disk that is tilted a few degrees out of the orbital plane and precessing slowly in the retrograde direction (Barrett et al. 1988; Foulkes et al. 2006; Wood \& Burke 2007, Wood et al. 2000, 2009). Instead of impacting the rim

of the disk, the accretion spot sweeps across first one face of the tilted disk and then the other during one orbit, impacting the rim only twice during one cycle, along the line of nodes. Thus, the accretion mass and energy is deposited throughout a range of radii in the disk, and this may act to stabilize the disk against DN outbursts. The slow rise of the first dwarf nova oscillations after the long quiescent state indicates that this is an inside-out outburst, consistent with the deposition of mass primarily in the inner disk. Clearly these data motivate further study of the thermal-viscous limit cycle instability in tilted disks.

The primary interpolation scheme we considered, given in eqn. (24), has clear problems in making good superoutbursts. The model superoutbursts have shoulders which comprise about a third to half of the total viscous plateau, rather than the $\sim 10 \%$ observed. However, 
for the H98 scaling with $T_{0}=12500 \mathrm{~K}$, the shoulders are appropriately short, $\sim 2 \mathrm{~d}$, and the viscous plateaus much longer. The rate of decay of the viscous plateau is too fast, but that is because the input parameters have been optimized for the scaling given in eqn. (24).

\section{Discussion}

In contrast to the thermal-tidal instability of Osaki (Ichikawa \& Osaki 1992, 1994; Ichikawa, Hirose, \& Osaki 1993) in which the tidal torque is artificially enhanced by a factor of 20 when the outer disk expands beyond the point of 3:1 commensurability with the orbital period, our detailed modeling shows that the regular and superoutbursts can produced with a constant set of parameters. In retrospect, one may legitimately question the motivation for the increased tidal torque accompanying the expansion of the disk, considering that the ultimate driving force behind the tidal torque is the gravitational field of the secondary, which remains unchanged. We share a common viewpoint with van Paradijs (1983) in that we regard the superoutbursts in the SU UMa systems as an extension of the long outbursts in DNe above the period gap, such as U Gem and SS Cyg (Cannizzo \& Mattei 1992, 1998; C93b). Continued monitoring of V344 Lyr should reveal the stability of the long term sequencing, i.e., variations in the number of shorts sandwiched between two longs.

The shoulders on the rise to superoutburst in the models have more structure than the shoulder in the observed superoutburst. The shoulders arise because the initial tendency of the triggering of thermal instability is to produce a short outburst. However, due to the long-term net build-up of mass in the disk at large radii between successive short outbursts, there is actually enough material accumulated to support a superoutburst. It takes a little longer however for the outward moving heating front to progress to larger radii and to fully access this additional store of matter, which manifests itself as a ring of enhanced surface density in the outer disk. This explains the fact that many observed superoutbursts appear to start as normal (short) outbursts. The subsequent rise after the shoulder is due to the full radial extent of the disk out to the outer edge making a transition to the hot state. In contrast, for the short (normal) outbursts, not enough disk mass has accumulated at larger radii by the time of the outburst to support the heating transition front propagating to large radii, therefore the heating front stalls at some $r<r_{\text {outer }}$ and is reflected as a cooling front, leading to pointy maxima rather than viscous plateaus.

Our testing has delineated a range of allowable parameters:

(1) The logarithmic interpolation for $\alpha$ produces well-separated outbursts, with concave downward decays, as observed. The linear interpolation produces outbursts with minimal 
quiescence and concave upward decays. Given the physical underpinnings of the MRI, the logarithmic interpolation is better motivated since the MRI strength should depend on the partial ionization fraction, which is a strongly increasing function of temperature.

In terms of the superoutburst, the H98 scaling with $T_{0}=12500 \mathrm{~K}$ produces the best overall results. The shoulder feature only persists $\sim 2 \mathrm{~d}$, as observed, and the viscous plateau is longer. About $70 \%$ of the stored mass is accreted. The main deficiencies are that the fast decay (i.e., that following the viscous plateau) is slower-than-exponential, and the rise times are too fast (as with the other calculations).

(2) The minimum number of grid points necessary is $\gtrsim 10^{3}$ (in accord with C93b and H98). Even for high $N$, the sequencing is not perfectly stable.

(3) Of the terms entering into the thermal energy equation, the radial viscous transport term $C$ and the second advective term, $v_{r}(\partial T / \partial r)$ appear to be the most important. The radial radiative flux term $D$ and the first advective term, involving $\partial\left(r v_{r}\right) / \partial r$, are of lesser importance.

(4) The values for $\alpha_{\text {cold }}=0.0025, \alpha_{\text {hot }}=0.1$, and $\dot{M}_{T}=2 \times 10^{-10} M_{\odot} \mathrm{yr}^{-1}$ are mandated by the observed spacing and duration of short outbursts and the superoutburst. Increasing their values decreases the outburst time scales. The inferred values for $\alpha_{\text {cold }}$ and $\alpha_{\text {hot }}$ are to be viewed merely as best-fit parameters for V344 Lyr; they do not allow us to draw any deep conclusions as regards other systems.

(5) The values $r_{\text {inner }}$ and $r_{\text {outer }}$ strongly mediate the number of short outbursts between two superoutbursts. For $N=800$ and $f_{t}=1 / 160$ we have $\sim 5$ shorts between two longs for $r_{\text {inner }}=2 \times 10^{9} \mathrm{~cm}$, or $\sim 3 R_{\mathrm{WD}}$ for $M_{\mathrm{WD}}=0.6 M_{\odot}$. Observations by Howell et al. (1999) seem to favor an evacuated inner disk. Several possibilities are discussed by these workers, including the possibility of weak intermediate polars in the SU UMa systems. The outbursts in our models are triggered at intermediate disk radii, therefore the outer disk radius has minimal effect on the outburst recurrence times and durations. Increasing $r_{\text {outer }}$ from $1 \times 10^{10}$ $\mathrm{cm}$ to $2 \times 10^{10} \mathrm{~cm}$ increases the number of shorts between to successive longs, and also distorts the profile of the superoutburst.

With $\alpha_{\text {hot }}=0.1$, we match the rate of decay of the viscous portion of the superoutburst, and also find a $\sim 0.5$ mag shoulder on the rise to superoutburst. It is not clear why previous detailed time dependent modelers did not find this shoulder (e.g., S04). The main deficiency in the models with the standard logarithmic $\alpha_{\text {cold }} \leftrightarrow \alpha_{\text {hot }}$ interpolation is their failure to produce a sufficiently long viscous plateau on the superoutburst. The observed plateau has a decay rate of $\sim 12 \mathrm{~d} \mathrm{mag}^{-1}$ and lasts for $\sim 12 \mathrm{~d}$, which corresponds to $\sim 1 e$-folding in the decay of the disk mass, meaning about $60 \%$ of the disk mass should be accreted. Our viscous 
plateau lasts $\sim 6-8 \mathrm{~d}$, with proportionally less mass accreted. However, the light curve using the H98 scaling with $T_{0}=12500 \mathrm{~K}$ (which appears to be physically motivated insofar as it tracks $\xi$ ) has substantially longer superoutbursts, $\sim 10 \mathrm{~d}$, with appropriately short shoulders as observed. The reason for the shorter shoulders is that the temperature within the outward moving heating front accompanying superoutburst onset is between about $10^{4}$ and $2 \times 10^{4}$ K. As can be seen in Figure 5 by comparing the left-most green curve (i.e., the H98_12500 scaling) with the "log" curve, for the temperature range $10^{4} \mathrm{~K}<T<2 \times 10^{4} \mathrm{~K}$, $\alpha$ is larger in the H98_12500 scaling and therefore the heating front travels faster since its speed varies with $\alpha$ (Menou, Hameury, \& Stehle 1999). The duration of the shoulder feature corresponds to the interval during which the heating front is moving outward and finding that enough mass is present at large radii to support a superoutburst. This causes the dip and subsequent rise to the plateau of the superoutburst.

The values for $\alpha_{\text {cold }}, \alpha_{\text {hot }}$, and $\dot{M}_{T}$ have already been optimized to get the supercycle and normal cycle periods of $\sim 100 \mathrm{~d}$ and $\sim 10 \mathrm{~d}$, respectively. One suspects that the scalings for $\Sigma_{\max }\left(\alpha_{\text {cold }}\right)$ and $\Sigma_{\min }\left(\alpha_{\text {hot }}\right)$ are too simplistic and fail to take into account the actual disk physics, particularly that of the quiescent state, which is presently unknown. One could envision introducing an ad hoc multiplicative parameter for $\Sigma_{\max }$, for instance to artificially enhance it, but then one would have to re-adjust all the other parameters, in particular increasing $\dot{M}_{T}$ which is already uncomfortably large. In view of these considerations we consider our experiment moderately successful in that we are able to reproduce the duration of the viscous plateau within a factor of two in an over-constrained system for which $\alpha_{\text {cold }}$ and $\alpha_{\text {hot }}$ have already been determined by the outburst properties.

What about having enhanced mass transfer from the secondary prolong the duration of the superoutburst? Smak (2004) presented observational evidence for enhanced hot spot brightness near superoutburst maxima which he interpreted as being due to enhanced mass transfer brought about by the strong irradiation of the secondary. Osaki \& Meyer (2003) presented a theoretical study of the effects of irradiation on the photosphere of the secondary star in SU UMa systems and concluded that mass transfer could not be enhanced by a significant amount because the Coriolis force prevents the formation of a circulation flow transporting heat toward L1. Viallet \& Hameury $(2007=$ V07, 2008=V08) present two-dimensional, time dependent calculations of the surface flow of material of irradiated secondaries and find the physical situation to be significantly different from that envisioned by either Osaki \& Meyer or Smak. The Coriolis force leads to the formation of a circulation flow from the high latitude regions to the vicinity of the L1 point, but rapid cooling of the gas as it enters the shielded equatorial region prevents significant heat transport (V07). V07 conclude that any resulting mass transfer rate enhancement is likely to be moderate and unable to account for the duration of long outbursts in dwarf novae. V08 look at two effects that could potentially 
induce secondary mass transfer rate variations in dwarf novae and soft X-ray transients: irradiation of the secondary by the hot outer disk rim during outburst, and scattered radiation by optically thin outflowing material. They conclude that for dwarf nova parameters the effects on enhanced mass transfer are negligible. For completeness we note that some of the assumptions of V07 and V08 may not apply if the disk is warped or tilted, in which case the L1 point could in principle be directly irradiated.

S04 presented detailed time dependent calculations of the accretion disk evolution in both the TTIM and the EMTM, and in the end concluded that the EMTM was favored. Their arguments are rather general, however, and they state "... we have not proven the EMTM to be correct nor the TTIM not to work." Both models have a number of free parameters in addition to those already present in the standard limit cycle model. For the TTIM one has the time delay between the attainment of the 3:1 radius by the outer disk edge and the onset of enhanced tidal torque, in addition to the functional form and amplitude associated with the increase. Similarly the EMTM has as free parameters the degree of augmentation of $\dot{M}_{T}$ during superoutburst, as well as its functional form with time.

In addition, there may be a problem with the viscous decay in the EMTM: The V344 Lyr superoutburst maintained a closely exponential decay over $\sim 1$ mag, whereas our models as well as others reveal a definite slower-than-exponential trend (i.e., concave upward when plotted as magnitude versus time) in viscous plateaus covering that much dynamic range. Although the viscous decays can be lengthened in the EMTM, they tend to have noticeable deviations from exponentiality, in contrast to the V344 superoutburst. The fact that the short orbital period system WZ Sge did show a deviation from exponentiality in its 2001 outburst, which also spanned a greater dynamic range than in the V344 Lyr superoutburst

( $\sim 2$ mag versus $\sim 1 \mathrm{mag}$ ), may indicate that irradiation-induced enhanced mass transfer is a factor at much shorter orbital periods than for V344 Lyr. In addition, the amplitude of the $\sim 0.5 \mathrm{mag}$ shoulder on the rise to superoutburst is roughly matched in our calculations; with enhanced mass transfer the shoulder amplitude is too great ( $\gtrsim 1 \mathrm{mag}$ ). We consider it fortunate that we have matched the decay as well as we have since the scalings for the extrema in the limit cycle model, in particular $\Sigma_{\max }\left(\alpha_{\text {cold }}\right)$, are most probably gross simplifications to a more complicated situation that is currently beyond our understanding.

\section{Conclusion}

We have presented detailed time dependent calculations of the accretion disk limit cycle mechanism with application to the Kepler light curve of V344 Lyr. We find that the basic disk instability model is able to account for the mixture of normal and superoutbursts with 
a minimal amount of fine-tuning. Given the additional free parameters associated with both the thermal-tidal model and the enhanced mass transfer models, we do not attempt to examine their possible ramifications in this work. It may be that the plain vanilla disk instability acting alone is sufficient to explain most aspects of the long term V344 Lyr light curve. We also note that the normal outbursts in V344 Lyr do not show a monotonic increase in recurrence time and amplitude between successive superoutbursts as predicted by the TTIM (see Ichikawa, Hirose, \& Osaki 1993, Fig. 1; Osaki 2005, Fig. 3), but rather a maximum in these quantities near the middle of a supercycle. The models we present also do not show the observed variations.

One can in some sense view the alternation of many successive short outbursts with a superoutburst as an extensive of the pattern of short and long outbursts seen in the longer orbital period systems such as U Gem and SS Cyg (van Paradijs 1983); for SU UMa parameters one has many more short outbursts between two longs than in a system like SS Cyg, which exhibits LSLSLS... most of the time (Cannizzo \& Mattei 1992). For our best-fit model parameters, we find that $\gtrsim 800$ grid points are required for numerical stability, with a resulting pattern in which 5-7 short outbursts occur between two successive superoutbursts (for $f_{t}=1 / 640$ ). Overall, our outbursts are at about the same brightness as those observed in V344 Lyr for an intermediately inclined disk. Having constrained $\alpha_{\text {cold }}$ and $\alpha_{\text {hot }}$ so as to produce the overall recurrence time scales of short outbursts and superoutbursts, there are several obvious deficiencies: the rise times of all outbursts are too fast, the superoutburst duration is too short, and the duration of the shoulder is too long and has an extra scalloped-out feature prior to the observed shoulder. However, the more physically motivated H98 scaling with $T_{0}=12500 \mathrm{~K}$ does a better job on making superoutbursts with minimal shoulders as observed, lasting $\sim 2 \mathrm{~d}$, and also longer viscous plateaus, $\sim 10 \mathrm{~d}$ vs. the observed $\sim 12 \mathrm{~d}$. The normal outburst embedded at the start of a superoutburst is due to the initial triggering of the thermal instability and outward movement of the heating front; the subsequent dip and rise to superoutburst maximum comes about because the heating front continues to move outward, and subsequently to access the stored material at large radii in the disk which accumulates where the heating front spike stalls at the end of a normal outburst.

Even as we struggle to account for the finer points of the light curve, such as the shoulder on the superoutburst, we feel compelled to step back briefly from our myopic scrutiny of the details and give some modicum of thanks and appreciation for the fact that the Kepler data represent such a huge advance over what was available even as recently as one year ago. For long term dwarf nova light curves, the only data sets that had been available were those amassed by the AAVSO and similar organizations. While these data have certainly been useful and yielded many interesting results, one could only study in detail the brightest 
systems, like SS Cyg and U Gem. In addition, the data quality was uneven and the time sampling coarse. When new data become available that are an order of magnitude better than the previous data, it becomes possible to make discoveries that are different in "kind", not just in "degree". The Kepler data will provide a physical touchstone for modelers with the potential to substantially improve our understanding of the physics of accretion disks; our work represents only the first small step in this regard.

We thank James Bubeck (under the SESDA II contract) at Goddard Space Flight Center for assistance in providing local computational resources, and Marcus Hohlmann and Patrick Ford from the FIT High Energy Physics group and the Domestic Nuclear Detection Office in the Dept. of Homeland Security for making additional resources on a Linux cluster available.

We also thank the anonymous referee whose comments helped improve and clarify the paper. We acknowledge the contributions of the entire Kepler team. 


\section{References}

Ak, T., Bilir, S., Ak, S., \& Ezer, Z. 2008, New Astr, 13, 133

Ak, T., Ozkan, M. T., \& Mattei, J. A. 2002, A\&A, 389, 478

Angelini, L., \& Verbunt, F. 1989, MNRAS, 238, 697

Balbus, S. A., \& Hawley, J. F. 1998, Rev Mod Phys, 70, 1

Barrett, P., O’Donoghue, D., \& Warner, B. 1988, MNRAS, 233, 759

Bath, G. T., \& Pringle, J. E. 1981, MNRAS, 194, 967

Buat-Ménard, V., \& Hameury, J.-M. 2002, A\&A, 386, 891

Buat-Ménard, V., Hameury, J.-M., \& Lasota, J.-P. 2001, A\&A, 366, 612

Cannizzo, J. K. 1993a, in Accretion Disks in Compact Stellar Systems, ed. J. C. Wheeler (Singapore: World Scientific), 6 (C93a)

Cannizzo, J. K. 1993b, ApJ, 419, 318 (C93b)

Cannizzo, J. K. 1994, ApJ, 435, 389

Cannizzo, J. K. 1998a, ApJ, 493, 426

Cannizzo, J. K. 1998b, ApJ, 494, 366

Cannizzo, J. K. 2001a, ApJ, 556, 847

Cannizzo, J. K. 2001b, ApJ, 561, L175

Cannizzo, J. K., Gehrels, N., \& Mattei, J. A. 2002, ApJ, 579, 760

Cannizzo, J. K., \& Mattei, J. A. 1992, ApJ, 401, 642

Cannizzo, J. K., \& Mattei, J. A. 1998, ApJ, 505, 344

Cannizzo, J. K., Shafter, A. W., \& Wheeler, J. C., 1988, ApJ, 333, 227 (CSW)

Cannizzo, J. K., \& Wheeler, J. C., 1984, ApJS, 55, 367

Cannizzo, J. K., Wheeler, J. C., \& Polidan, R. S. 1986, ApJ, 301, 634 (C86)

Faulkner, J., Lin, D. N. C., \& Papaloizou, J. 1983, MNRAS, 205, 359

Foulkes, S. B., Haswell, C. A., \& Murray, J. R. 2006, MNRAS, 366, 1399

Fromang, S., \& Papaloizou, J. 2007, A\&A, 476, 1113 
Hameury, J.-M. 2002, in The Physics of Cataclysmic Variables and Related Objects, ed. B. T. Gänsicke, K. Beuermann, \& K. Reinsch (San Francisco; ASP), 377

Hameury, J.-M., Menou, K., Dubus, G., Lasota, J.-P., \& Hure, J.-M. 1998, MNRAS, 298, 1048 (H98)

Harrison, T. E., Johnson, J. J., McArthur, B. E., Benedict, G. F., Szkody, P., Howell, S. B., \& Gelino, D. M. 2004, AJ, 127, 460

Harrison, T. E., McNamara, B. J., Szkody, P., McArthur, B. E., Benedict, G. F., Klemola, A. R., \& Gilliland, R. L. 1999, ApJL, 515, L93

Hirose, S., Krolik, J. H., \& Blaes, O. 2009, ApJ, 691, 16

Hoffmeister, C., 1966, Astron. Nachr., 289, 139

Howell, S. B., Ciardi, D. R., Szkody, P., van Paradijs, J., Kuulkers, E., Cash, J., Sirk, M., \& Long, K. S. 1999, PASP, 111, 342

Howell, S. B., Nelson, L. A., \& Rappaport, S. 2001, ApJ, 550, 897

Ichikawa, S., Hirose, M., \& Osaki, Y. 1993, PASJ, 45, 243

Ichikawa, S., \& Osaki, Y. 1992, PASJ, 44, 15

Ichikawa, S., \& Osaki, Y. 1994, PASJ, 46, 621

Kato, T. 1993, PASJ, 45, L67

Kato, T., Poyner, G., \& Kinnunen, T. 2002, MNRAS, 330, 53

King, A. R., Pringle, J. E., \& Livio, M. 2007, MNRAS, 376, 1740

Kolb, U., King, A. R., \& Ritter, H. 1998, MNRAS, 298, L29

Lasota, J.-P. 2001, New Astron. Rev., 45, 449

Lightman, A. P. 1974, ApJ, 194, 419

Lin, D. N. C., Papaloizou, J., \& Faulkner, J. 1985, MNRAS, 212, 105

Lubow, S. H. 1991a, ApJ, 381, 259

Lubow, S. H. 1991b, ApJ, 381, 268

Lubow, S. H., \& Shu, F. H. 1975, ApJ, 198, 383

Ludwig, K., \& Meyer, F. 1998, A\&A, 329, 559 
Menou, K., Hameury, J.-M., \& Stehle, R. 1999, MNRAS, 305, 79

Meyer, F. \& Meyer-Hofmeister, E. 1981, A\&A, 104, L10

Meyer, F. \& Meyer-Hofmeister, E. 1982, A\&A, 106, 34

Meyer, F. \& Meyer-Hofmeister, E. 1984, A\&A, 132, 143

Meyer, F. \& Meyer-Hofmeister, E. 1994, A\&A, 288, 175

Mineshige, S. 1986, PASJ, 38, 831 (M86)

Mineshige, S. 1987, Ap \& Sp. Sci., 130, 331 (M87)

Mineshige, S. \& Osaki, Y. 1983, PASJ, 35, 377 (M83)

Mineshige, S. \& Osaki, Y. 1985, PASJ, 37, 1 (M85)

Mukai, K., Zietsman, E., \& Still, M. 2009, ApJ, 707, 652

Osaki, Y. 1989a, in Theory of Accretion Disks, ed. F. Meyer et al. (Kluwer: Dordrecht), 183

Osaki, Y. 1989b, PASJ, 41, 1005

Osaki, Y. 2005, Proc. Japan Acad., 81, 291

Osaki, Y., \& Meyer, F. 2003, A\&A, 401, 325

Papaloizou, J., \& Pringle, J. E. 1977, MNRAS, 181, 441

Pringle, J. E. 1981, ARA\&A, 19, 137

Pringle, J. E., Verbunt, F., \& Wade, R. A. 1986, MNRAS, 221, 169 (P86)

Schreiber, M. R., Hameury, J.-M., \& Lasota, J.-P. 2003, A\&A, 410, 239

Schreiber, M. R., Hameury, J.-M., \& Lasota, J.-P. 2004, A\&A, 427, 621 (S04)

Schreiber, M. R., \& Lasota, J.-P. 2007, A\&A, 473, 897

Shakura, N. I., \& Sunyaev, R. A. 1973, A\&A, 24, 337

Simpson, J. C., \& Wood, M. A. 1998, ApJ, 506, 360

Sion, E. M. 1995, ApJ, 438, 876

Smak, J. 1984, Acta Astr., 34, 161 (S84)

Smak, J. 1993, Acta Astr., 43, 101 
Smak, J. 2004, Acta Astr., 54, 221

Smak, J. 2009a, Acta Astr., 59, 89

Smak, J. 2009b, Acta Astr., 59, 103

Smak, J. 2009c, Acta Astr., 59, 109

Smak, J. 2009d, Acta Astr., 59, 121

Smak, J. 2010, Acta Astr., 60, 83

Still, M. R., Howell, S. B., Wood, M. A., Cannizzo, J. K., \& Smale, A. P. 2010, ApJL, 717, 113

van Paradijs, J. 1983, A\&AL, 125, L16

Viallet, M., \& Hameury, J.-M. 2007, A\&A, 475, 597

Viallet, M., \& Hameury, J.-M. 2008, A\&A, 489, 699

Vishniac, E. T. 2009, ApJ, 696, 1021

Warner, B. 1995a, Astrophys. \& Sp. Sci., 226, 187

Warner, B. 1995b, Cataclysmic Variable Stars (Cambridge: Cambridge Univ. Press)

Warner, B., \& O’Donoghue, D. 1988, MNRAS, 233, 705

Wheatley, P. J., Mauche, C. W., \& Mattei, J. A. 2003, MNRAS, 345, 49

Whitehurst, R. 1988, MNRAS, 232, 35

Wood, M. A., \& Burke, C. J. 2007, ApJ, 661, 1042

Wood, M. A., Montgomery, M. M., \& Simpson, J. C. 2000, ApJL, 535, L39

Wood, M. A., Thomas, D. M., \& Simpson, J. C. 2009, MNRAS, 398, 2110 
Table 1: Duration and Amplitude of Outbursts

\begin{tabular}{ccc}
\hline \hline Outburst & Duration (day) & Amplitude (mag) \\
\hline 1 & 4.9 & 4.0 \\
2 & 3.9 & 3.5 \\
3 & 4.4 & 3.6 \\
4 & $16.7(\mathrm{SO})$ & 4.5 \\
5 & 2.8 & 2.5 \\
6 & 3.0 & 2.6 \\
7 & 2.6 & 3.0 \\
8 & 3.6 & 3.5 \\
9 & 3.9 & 3.8 \\
10 & 3.8 & 3.7 \\
11 & 4.1 & 3.7 \\
12 & $17.1(\mathrm{SO})$ & 4.4 \\
13 & 3.8 & 2.7 \\
14 & 3.5 & 3.3 \\
15 & 3.6 & 3.5 \\
16 & 5.5 & 3.6 \\
17 & 4.1 & 3.8 \\
18 & 4.1 & 3.5 \\
19 & 4.3 & 3.5 \\
\hline
\end{tabular}




\section{Figure Captions}

Fig. 1. - A comparison of the Johnson $V$ band filter (red) with the Kepler filter (blue).

Fig. 2.- Kepler light curve of V344 Lyr over 270 d showing outbursts every $\sim 10$ d, with two superoutbursts. The flux is measured in $e^{-}$cadence $^{-1}$, where the cadence (time between integrations) is $1 \mathrm{~m}$. The full time resolution is shown. To obtain visual magnitude, a correspondence of $K p=12$ to $10^{7} e^{-}$cadence $^{-1}$ was adopted. (This conversion assumes an unreddened G2 star.)

Fig. 3.- The effect of using a logarithmic interpolation (eqn. 24) versus a linear one (eqn. 26]) for $\alpha$. For these runs $N=800$ and $f_{t}=1 / 160$. Shown are the light curves for the logarithmic case (top panel) and the linear case (second panel), the accompanying disk masses (third and fourth panels), and the radius of the hot/cold interface $r_{\mathrm{HC}, 10}$ during times when a transition front is present (fifth and sixth panels). For comparison the light curves for the disk-only contribution (i.e., excluding secondary and hot spot) are shown as dotted curves in the first and second panels. The disk masses are in units of $10^{22} \mathrm{~g}$ and $r_{\mathrm{HC}, 10}$ is in units of $10^{10} \mathrm{~cm}$.

Fig. 4.- The effect of using the H98 logarithmic factor $f$ (eqn. 25]). For these runs $N=800$ and $f_{t}=1 / 160$. Shown are the light curves for $T_{0}=25000 \mathrm{~K}$ (top panel), 18750 $\mathrm{K}$ (second panel), and $12500 \mathrm{~K}$ (third panel), the accompanying disk masses (fourth through sixth panels), and the radius of the hot/cold interface $r_{\mathrm{HC}, 10}$ during times when a transition front is present (seventh through ninth panels). The disk masses are in units of $10^{22} \mathrm{~g}$ and $r_{\mathrm{HC}, 10}$ is in units of $10^{10} \mathrm{~cm}$.

Fig. 5. - Shown are the different $\alpha_{\text {cold }}-\alpha_{\text {hot }}$ interpolations. The blue lines show the scalings for $T\left(\Sigma_{\max }\right)$ and $T\left(\Sigma_{\min }\right)$. The black curves indicate the linear (eqn. 26 ) and logarithmic (eqn. 24]) interpolations. The green curves show the H98 scaling (eqn. 25]), where the three curves (left to right) represent $T_{0}=25000 \mathrm{~K}, 18750 \mathrm{~K}$, and $12500 \mathrm{~K}$. The three red curves show a measure of the partial ionization; they are the relative contribution of electron pressure to total pressure as shown in Fig. 16 of $\mathrm{C} 93 \mathrm{~b}$, for $\rho\left(\mathrm{g} \mathrm{cm}^{-3}\right)=10^{-8}, 10^{-7}$, and $10^{-6}$ (left to right). The values given in C93b have been multiplied by $2 \alpha_{\text {hot }}$ to take into account the fact that (1) $P_{e} / P \rightarrow 0.5$ in the limit of complete ionization, and (2) in the high temperature limit $\alpha \rightarrow \alpha_{\text {hot }}=0.1$.

Fig. 6.- Model light curves showing the detail of a superoutburst for separate calculations in which $N=200$ (short dashed), 400 (long dashed), 800 (short dash-dotted), and 1600 (long dash-dotted). The green curve shows a superoutburst from the $T_{0}=12500 \mathrm{~K} \mathrm{H} 98$ scaling light curve of Fig. 4. For comparison the averaged Kepler data for the superoutbursts in 
Fig. 2 is shown (first: blue, second: magenta), where the original $\Delta t=1$ min cadence has been block-averaged to $\Delta t=4 \mathrm{hr}$ and a distance of $620 \mathrm{pc}$ was assumed in converting from $K p$ to $M_{\mathrm{Kp}}$. The two slanted red lines indicate a decay slope of $12 \mathrm{~d} \mathrm{mag}^{-1}$. The second panel shows the disk mass (in $10^{22} \mathrm{~g}$ ).

Fig. 7.- The effect of turning off different terms in the energy equation. For these runs $N=800$ and $f_{t}=1 / 160$. Shown are light curves using: the full equation (top panel), the turbulent transport $C=0$ (second panel), the radiative transport $D=0$ (third panel), both $C=0$ and $D=0$ (fourth panel), the first advective term set to zero (fifth panel), and the second advective term set to zero (sixth panel).

Fig. 8. - The effect of the inner disk radius. For these runs $N=800$ and $f_{t}=1 / 160$. The four panels show the resultant light curves for $r_{\text {inner }}=2 \times 10^{9} \mathrm{~cm}$ (top panel), $1.5 \times 10^{9} \mathrm{~cm}$ (second panel), $1 \times 10^{9} \mathrm{~cm}$ (third panel), and $0.5 \times 10^{9} \mathrm{~cm}$ (fourth panel).

Fig. 9.- The effect of the outer disk radius. For these runs $N=800$ and $f_{t}=1 / 160$. The six panels show the resultant light curves for $r_{\text {inner }}=0.8 \times 10^{10} \mathrm{~cm}$ (top panel), $1.0 \times 10^{10}$ $\mathrm{cm}$ (second panel), $1.25 \times 10^{10} \mathrm{~cm}$ (third panel), $1.5 \times 10^{10} \mathrm{~cm}$ (fourth panel), $1.75 \times 10^{10} \mathrm{~cm}$ (fifth panel), and $2.0 \times 10^{10} \mathrm{~cm}$ (sixth panel). 


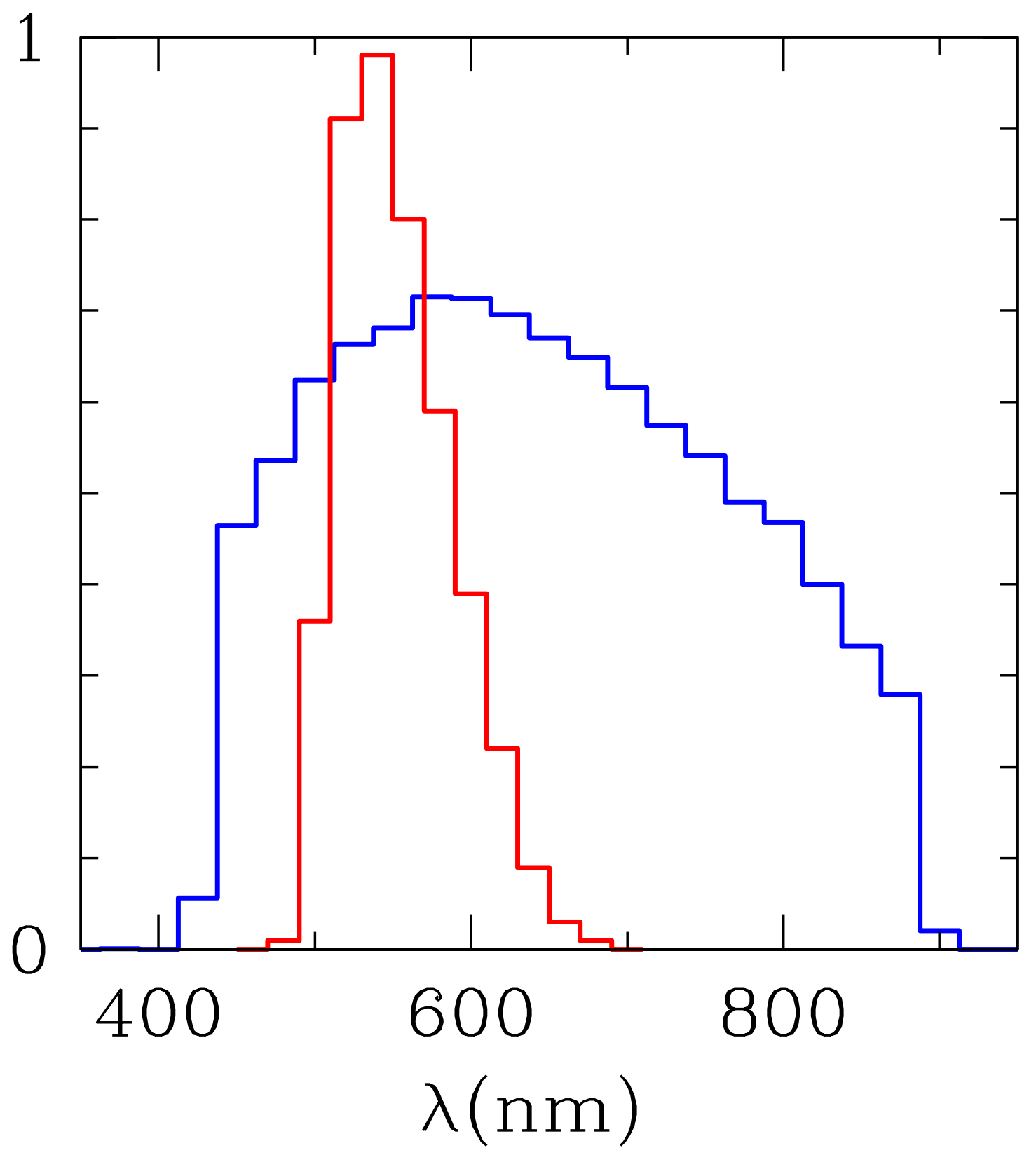




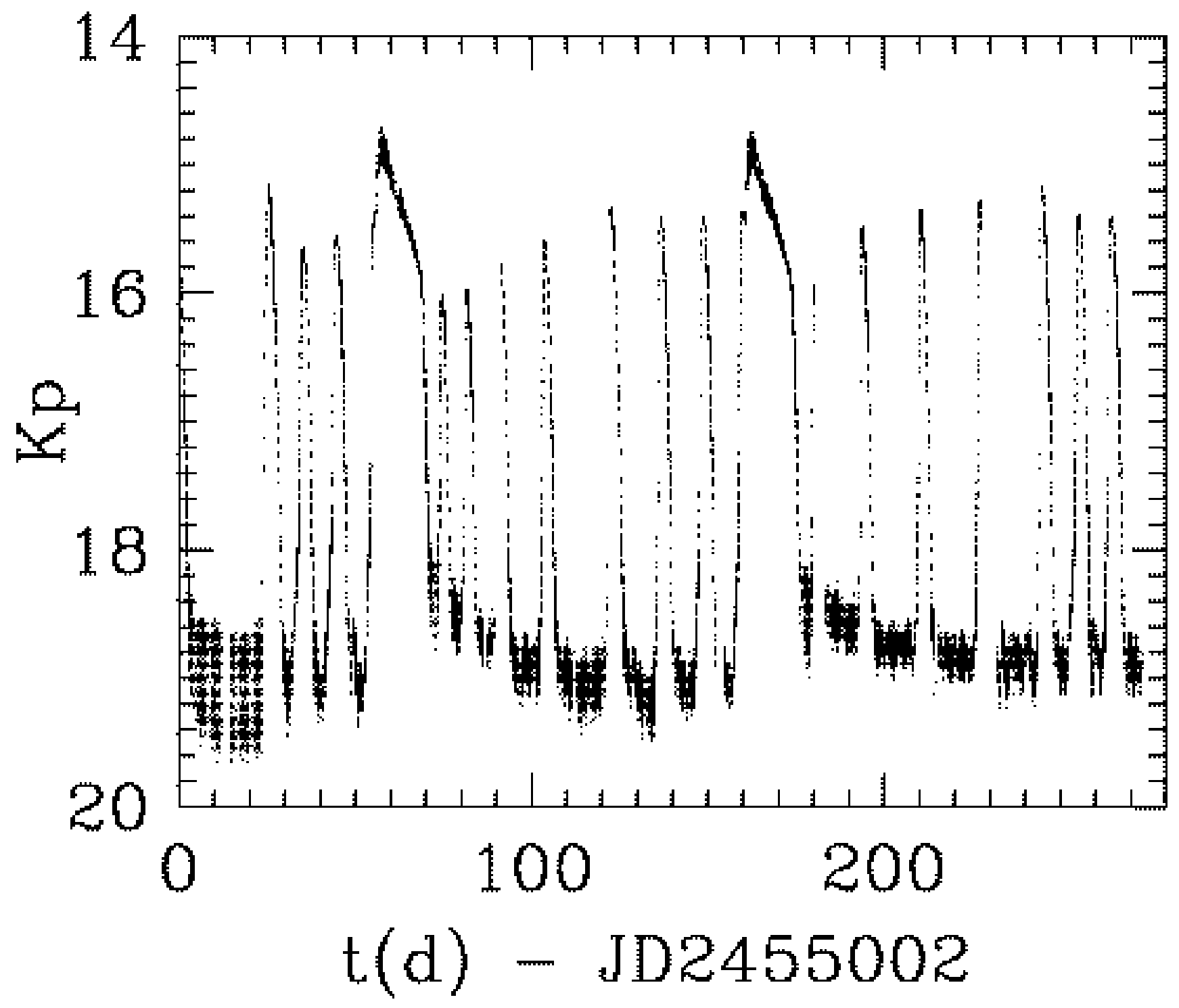




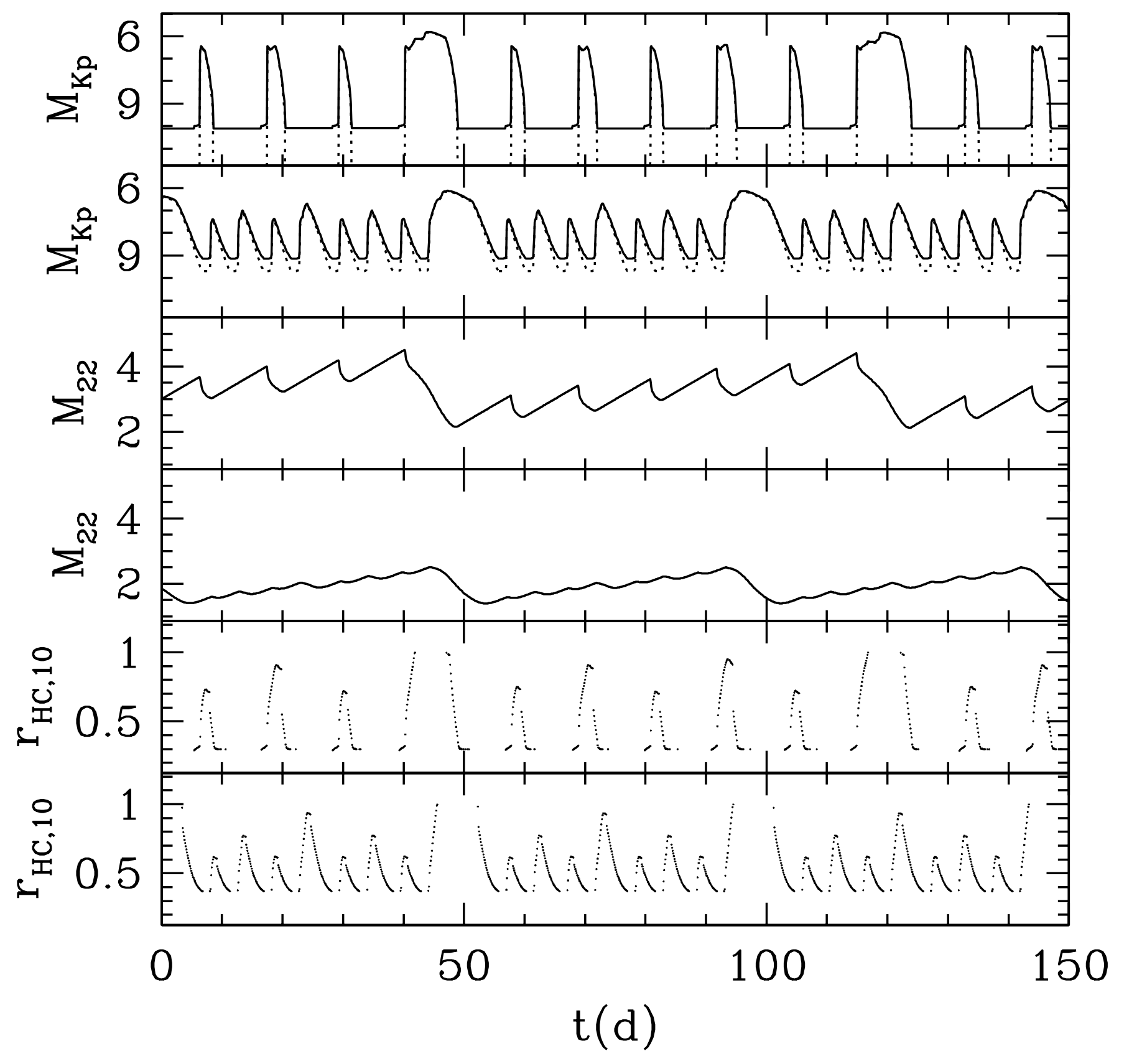




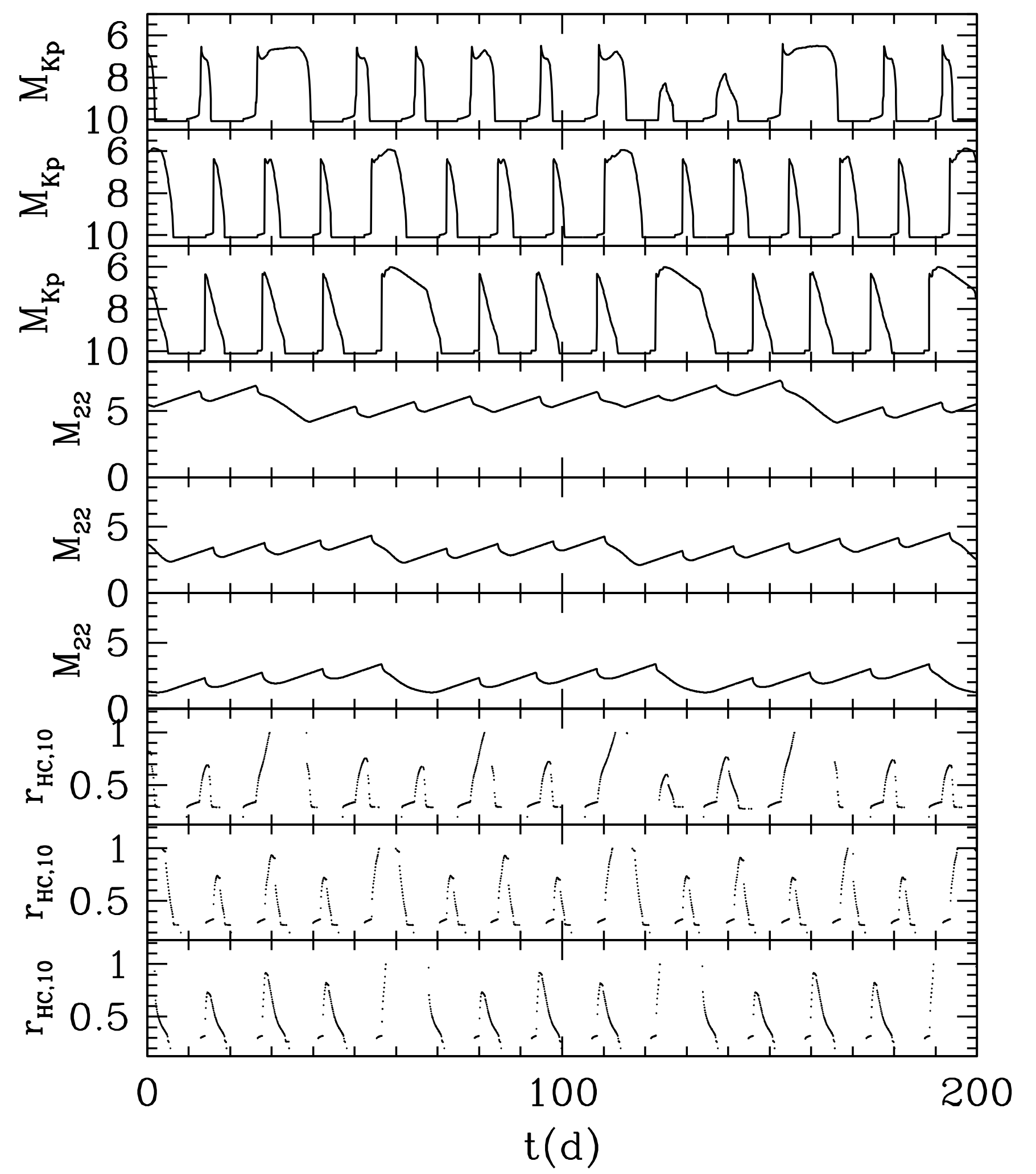




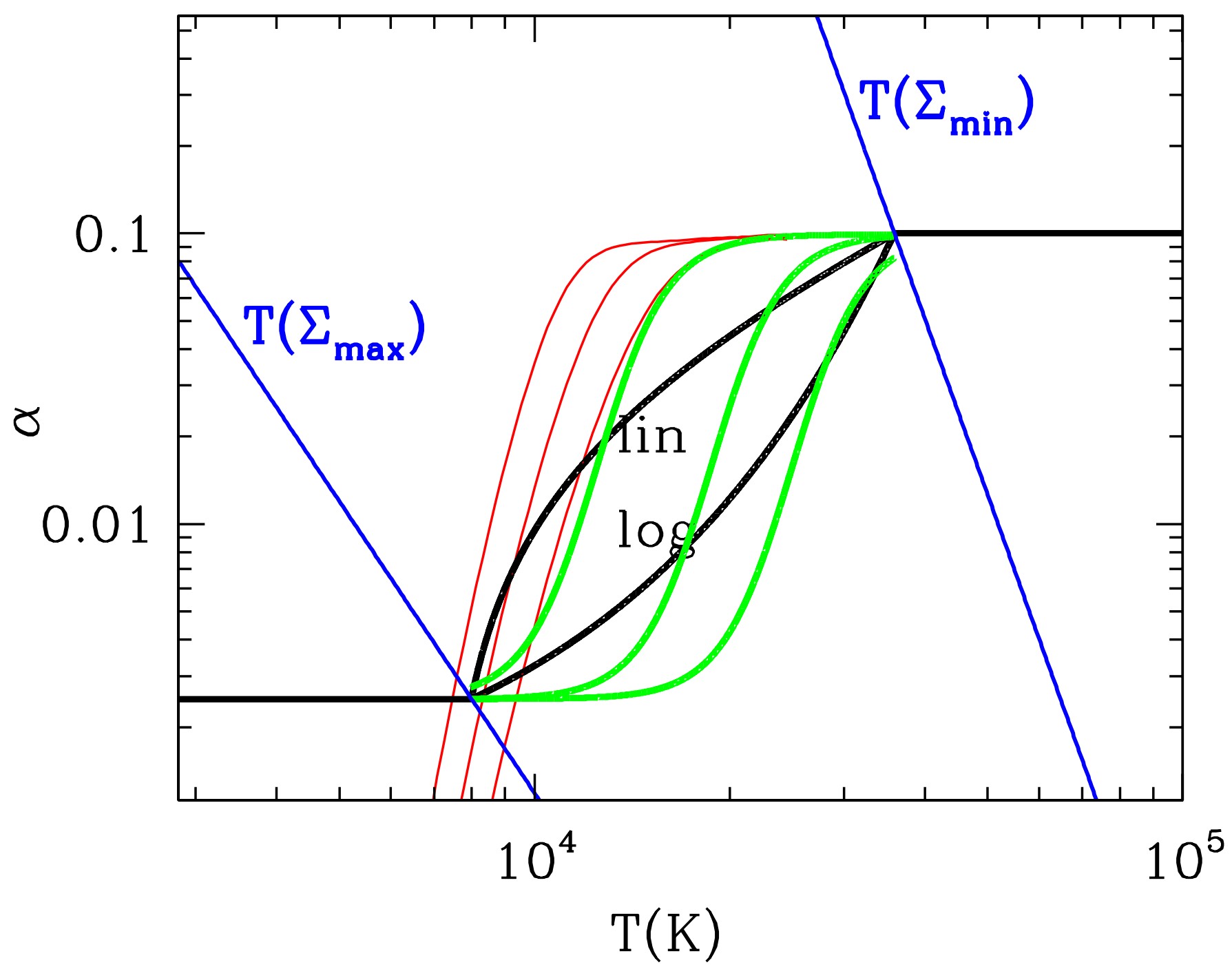

f5.ps 


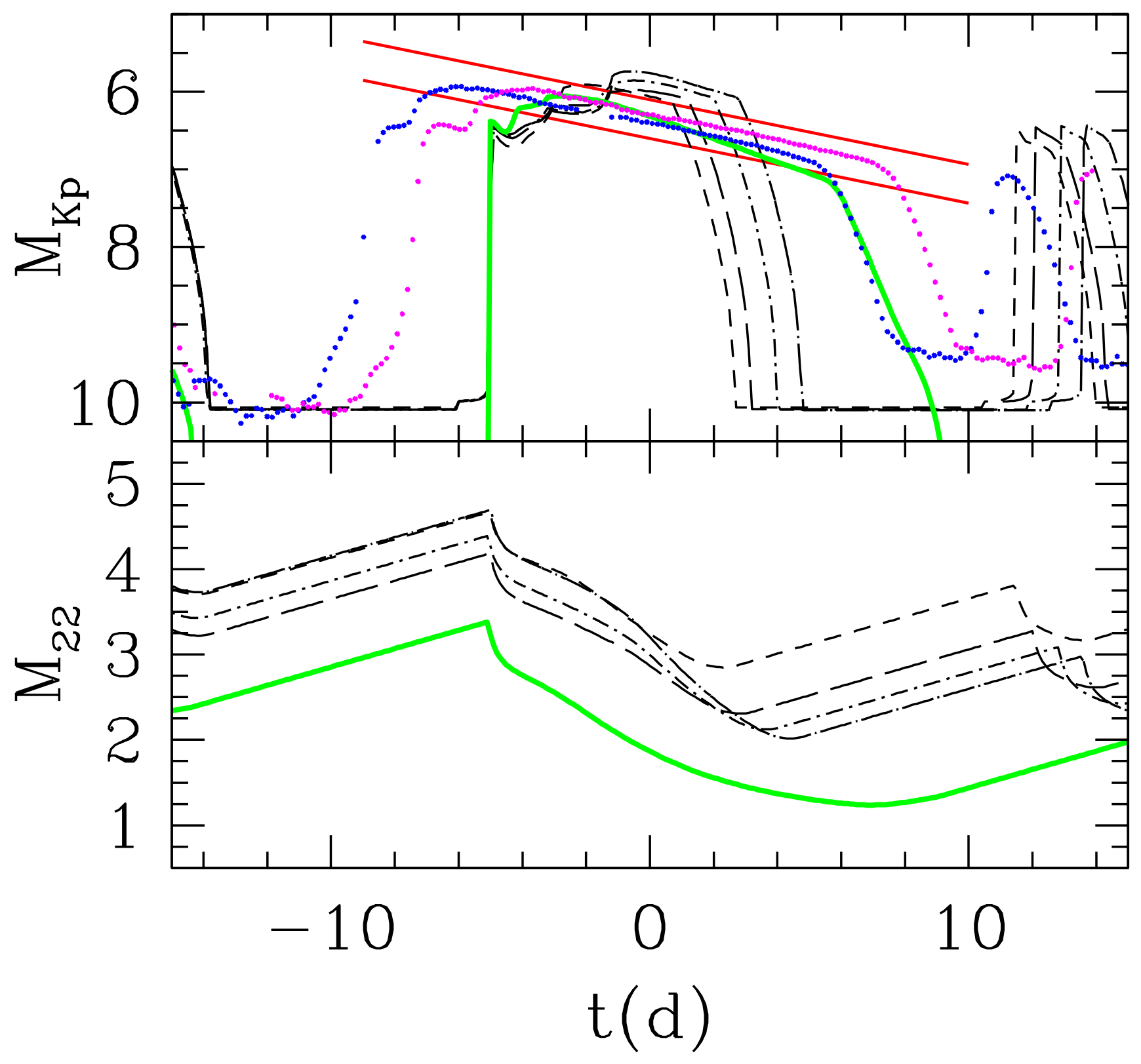

f6.ps 


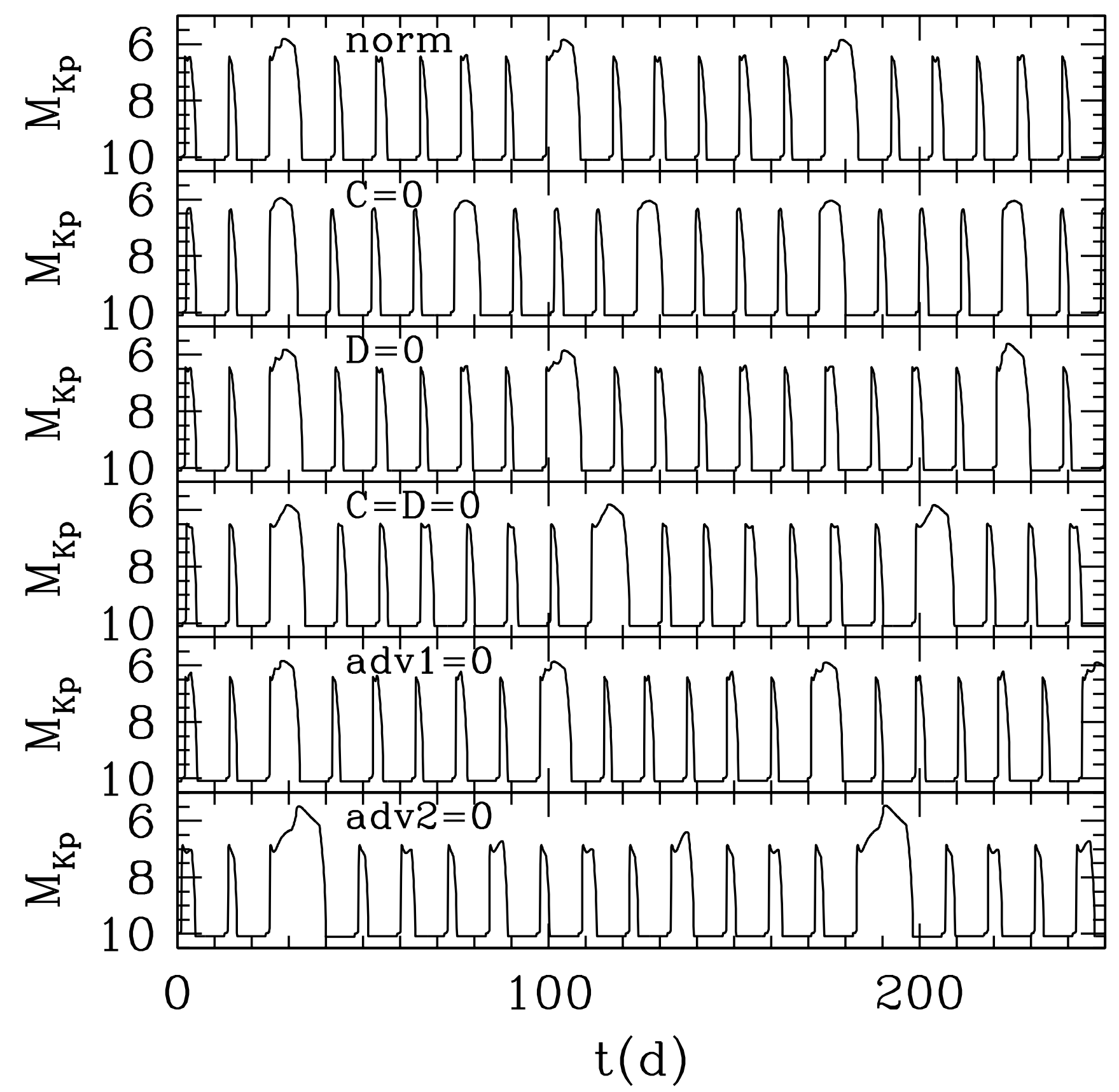




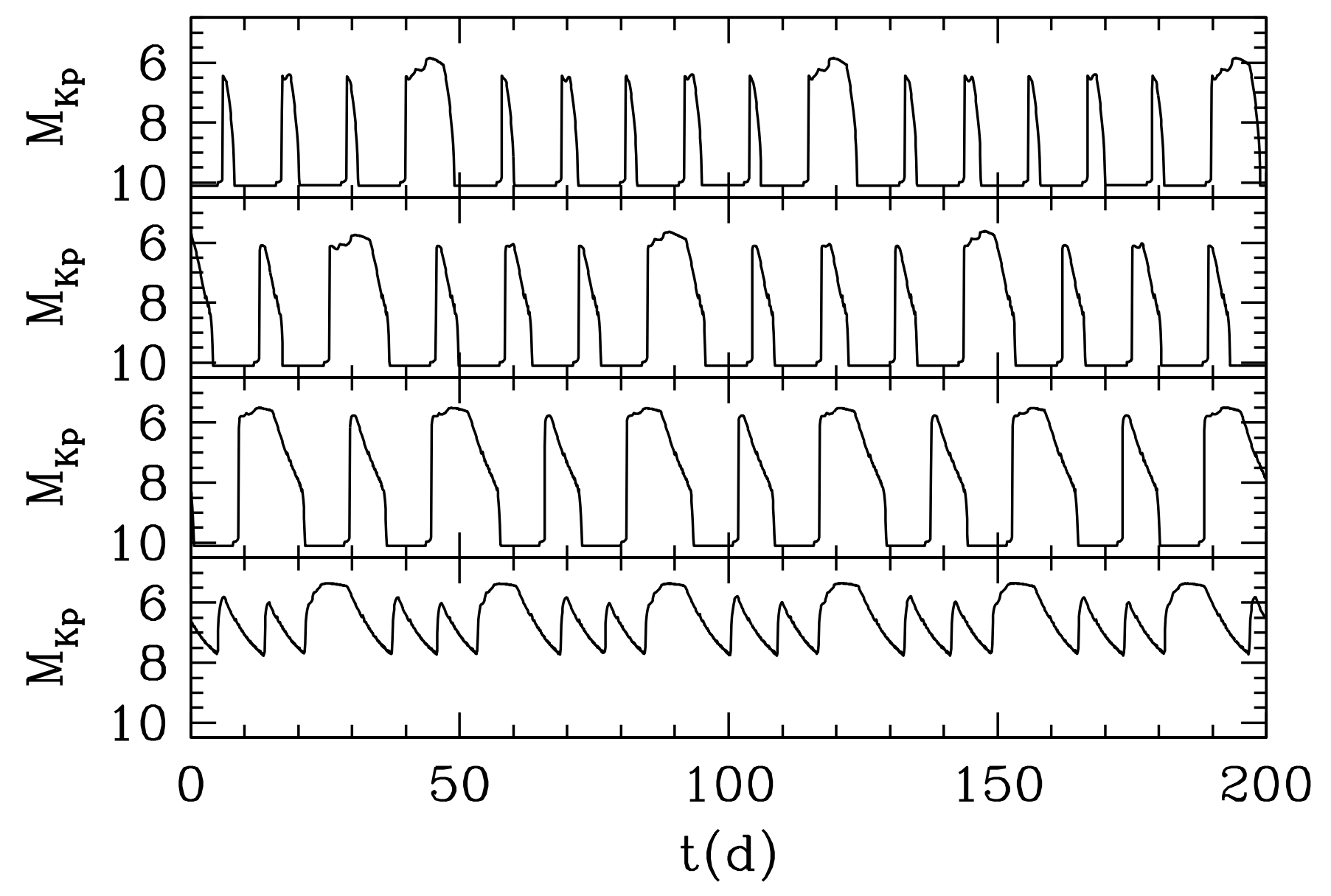




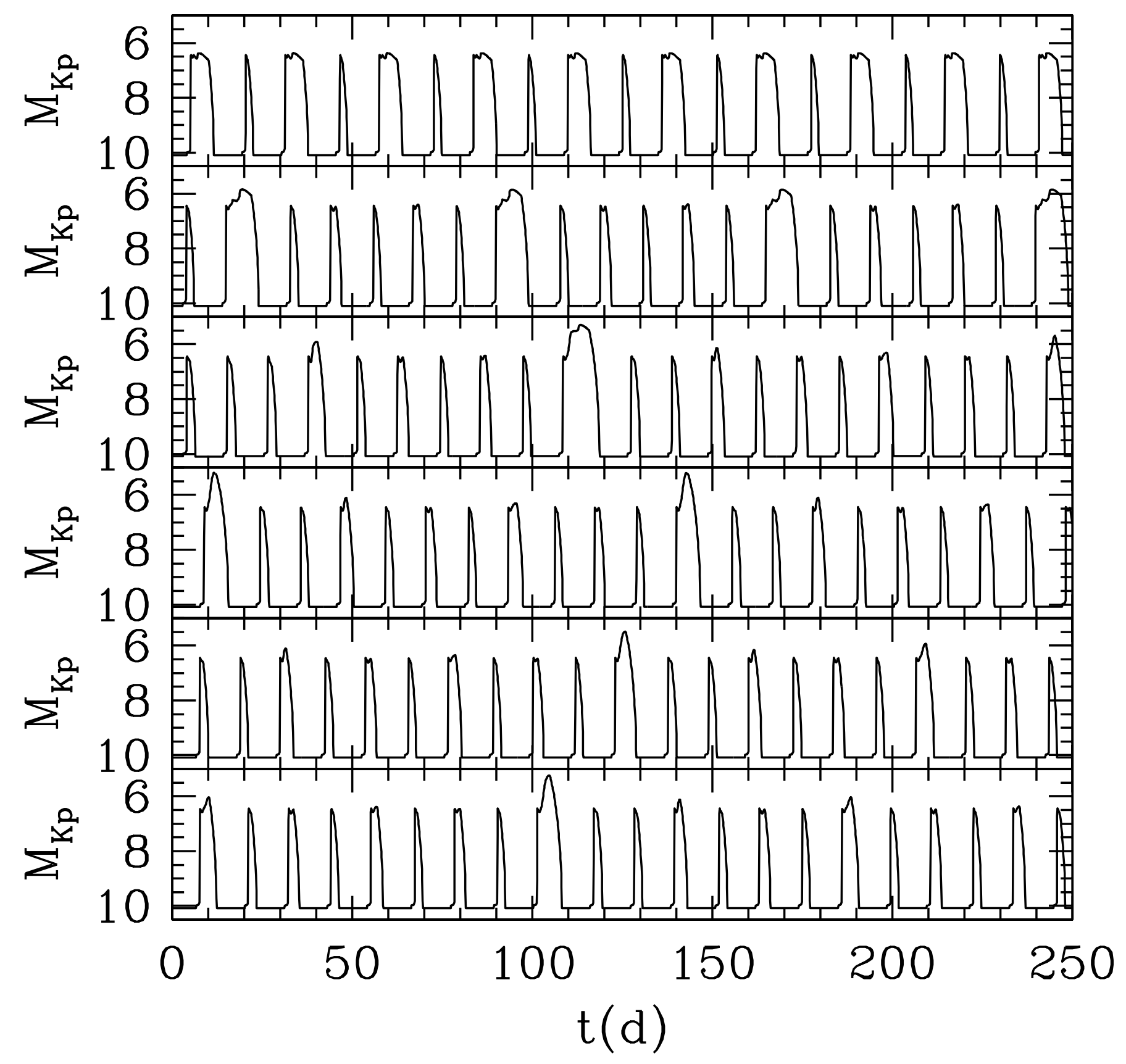

